\title{
Major and trace elements redistribution in weathered claystones from the Corumbataí Formation, Paraná Sedimentary Basin, São Paulo, Brazil
}

\author{
Redistribuição de elementos maiores e traços em \\ argilitos intemperizados da Formação Corumbataí, \\ Bacia Sedimentar do Paraná, São Paulo, Brasil
Letícia Hirata Godoy ${ }^{1 *}$, Diego de Souza Sardinha², Maria Margarita Torres Moreno ${ }^{1}$

\begin{abstract}
We studied 30 samples from 10 mining fronts of clayish rocks of the Corumbataí Formation, in the region of the Santa Gertrudes Ceramic Pole (São Paulo, Brazil), in order to evaluate the variation of rare earth elements (REE), mineralogy, weathering effects and provenance. The profiles show diagnostic geochemical signatures that are uniform across the Corumbataí Formation samples in the studied region and can be correlated from mine to mine, i.e.: 1) the constant $\mathrm{MgO} / \mathrm{K}_{2} \mathrm{O}$ ratio and the variation of other oxides, which allow the identification of three main groups of samples; 2) similar groups of chemical index of weathering (CIW) and chemical index of alteration (CIA); 3) the immobile character of REE, Th, and Sc. The erosion/denudation processes were apparently the same among the studied profiles, allowing the identification of a similar weathering pattern among the mines, despite its location within the stratigraphic column. Mineralogical and geochemical data here reported suggest a dominant felsic source, but intermediate or mixed sources cannot be discarded. The chemical and mineralogical aspects observed are practically uniform along the studied profiles and mines, which allow them to be used as raw material by the industries of the Santa Gertrudes Ceramic Pole (SGCP) region.
\end{abstract}

KEYWORDS: weathering; remobilization; sedimentary rocks; geochemistry; Paraná Basin; ceramic.
RESUMO: Foram analisadas 30 amostras de 10 frentes de lavra sobre rochas argilosas da Formação Corumbataí, na região do Polo Cerâmico de Santa Gertrudes (São Paulo, Brazil), visando avaliar a variação de elementos terras raras (ETRs), mineralogia, efeitos do intemperismo e proveniência. Os perfis apresentam assinaturas geoquímicas características que são uniformes nas amostras da Formação Corumbataí na região de estudo e que sáo correlacionáveis entre as minas, isto é: 1) a razão constante de $\mathrm{MgO} / \mathrm{K}_{2} \mathrm{O}$ e a variação de outros óxidos, o que permite o reconhecimento de três grupos principais de amostras; 2) grupos similares de indices quimico de intempéries (CIW) e químico de alteração (CIA); 3) o caráter imóvel de ETRs, Th e Sc. Os processos de erosáoldenudação aparentemente foram os mesmos entre os perfis estudados, permitindo a identificação de um padrão de intemperismo similar entre as minas, independentemente de sua localização na coluna estratigráfica. Os dados mineralógicos e geoquimico aqui apresentados sugerem uma fonte dominantemente félsica, porém fontes intermediárias ou mistas não podem ser descartadas. Os aspectos químicos e mineralógicos observados são praticamente uniformes ao longo dos perfis e minas, o que permite sua utilização como matéria-prima para as indústrias da regiāo do polo cerâmico.

PALAVRAS-CHAVE: intemperismo; remobilização; rochas sedimentares; geoquímica; Bacia do Paraná; cerâmica.

\footnotetext{
${ }^{1}$ Departamento de Petrologia e Metalogenia, Instituto de Geociências e Ciências Exatas, Universidade Estadual Paulista "Júlio de Mesquita Filho" - UNESP, Rio Claro (SP),Brazil.E-mails: leticiahirata@gmail.com,mmoreno@rc.unesp.br

${ }^{2}$ Instituto de Ciência e Tecnologia, Universidade Federal de Alfenas - UNIFAL, Poços de Caldas (MG), Brazil. E-mail: diegosouzasardinha@gmail.com

*Corresponding author

Manuscript ID: 20170086. Received in: 03/28/2017. Approved in: 08/25/2017
} 


\section{INTRODUCTION}

The Santa Gertrudes Ceramic Pole (SGCP), located in São Paulo State, approximately $180 \mathrm{~km}$ from the capital, is the biggest ceramic center in Latin America. The raw material utilized by the ceramic industries in the region is considered great when compared to other sedimentary rocks, since its natural properties allow achieving good quality products in relatively low temperatures with the use of dry milling process, therefore reducing the energetic costs (Azzi et al. 2016).

The raw material is inserted in the geological context of Paraná Sedimentary Basin, a typically intracratonic basin, which is filled by sedimentary and basic igneous rocks, whose age varies from Late Ordovician to Late Cretaceus (Fúlfaro \& Bjornberg 1983; Milani et al. 1994).

In the region of the SGCP and vicinities, the lithostratigraphy of the Paraná Sedimentary Basin is represented by four main units, i.e., Itararé Group, Guatá Group (Tatuí Formation), Passa Dois Group (Irati and Corumbataí formations), and Sáo Bento Group (Piramboia, Botucatu and Serra Geral formations), from Late Carboniferous to Early Cretaceous, respectively. The raw material encompasses the Corumbataí Formation sedimentary rocks, whose lower and upper contacts are with Irati and Piramboia formations respectively, locally affected by the basic igneous dykes of the Serra Geral Formation.

The raw material exhibits distinctive physical-chemical characteristics due to heterogeneity as a result of geological processes that lead to rock consolidation and weathering processes. The mineralogy of the claystones from Corumbataí Formation is dominantly composed by illitic clays, but chlorite, smectite and sometimes kaolinite are common too, this last one in the upper levels. Also, quartz, carbonate, hematite, feldspar (mostly sodic), analcime and others, in minor proportions, occur along (Moreno et al. 2009; Moreno et al. 2014).

The Corumbataí Formation claystones in the studied region present a practically constant $\mathrm{MgO} / \mathrm{K}_{2} \mathrm{O}$ ratio along with $\mathrm{SiO}_{2}$ increase, which constitutes a particularity that can be used to identify/select the raw materials from the weathering profiles in order to control the composition of the ceramic masses and final products, as previously indicated by Moreno et al. (2014).

In this paper, we address four relevant questions inherited from previous geochemical studies, by evaluating the mineralogy and chemical composition of major and trace elements, including the rare earth elements (REE), of 10 mining fronts:

1. Do the profiles show fingerprints that can be correlated from mine to mine?

2. In which conditions the claystones were affected by weathering processes and how the elements are remobilized and concentrated throughout the profiles and mines?
3. How the erosion/denudation affected the Santa Gertrudes Ceramic Pole (SGCP) weathered claystones?

4. How many sources supply sediment to the system, and how did these sources affect the characteristics of the raw material used in the SGCP?

\section{METHODS}

Several field works were performed in order to collect information about rock type, structures, soil profiles, availability and mode of occurrence of the clay ore, as well as photos and samples from all mines.

The samples, extracted with an auger, constitute the representative and homogenized material that occurs in one bench of the respective mining front. The numerical sequence of samples within a single profile increases from lower to upper portions. The samples were dried in a muffle furnace with a constant temperature of $60^{\circ} \mathrm{C}$ for 24 hours and dry milled in a hammer mill (Servitech, Santa Catarina, Brazil) coupled with a $1 \mathrm{~mm}$ sieve, in order to achieve a better homogenization.

The mineralogical analysis by X-Ray Diffractometry (XRD; Siemens/Bruker D5000, Wisconsin, USA) was measured with a copper radiation $(\mathrm{WL}=1,542 \AA$ ) and a nickel filter in a $2 \theta$ range of 3 to $65^{\circ}$. The goniometer velocity was set in $3^{\circ}(2 \theta)$ per minute, and an exposure time of 1 second for each $0,05^{\circ}$ step was set. The analysis was performed in four conditions: total sample; natural fine fraction; fine fraction treated with ethylene glycol; and fine fraction after fire at $500^{\circ} \mathrm{C}$ for 4 hours (Thiry et al. 2013). The diffractograms were analyzed in the X'Pert High Score Plus software.

The chemical analyses of major and trace elements, including REE, were performed in Acme Labs (Canada) with an inductively coupled plasma mass spectrometer (ICP-MS), using $0.2 \mathrm{~g}$ of sample and the lithium metaborate/tetraborate fusion and diluted nitric acid digestion technique. Binary and ternary variation diagrams were elaborated from this data, in order to understand the distribution of trace elements, including REE, in the study area as a whole and along each profile in different mining fronts.

\section{STUDY AREA}

A total of 30 samples were collected in 10 mining fronts located in the municipalities that comprehend the SGCP (Santa Gertrudes, Ipeuna, Rio Claro, Araras, Cordeirópolis and Limeira) and are inserted in the geological context of the Paraná Sedimentary Basin, as shown in Figures 1 and 2.

The clayish sedimentary rocks, used as raw material by the ceramic industries of the SGCP, belong to the Corumbataí 
Formation and were originated in a shallow marine environment transitioning to a fluvial-lacustrine continental environment (Milani et al. 1994). Basic igneous dikes of the Serra Geral Formation are related to a magmatic province that feature flows and intrusions of tholeitic basalts (White 1908). Zanardo et al. (2016) suggest that the Corumbataí Formation was subjected to thermal effect after lithification, promoted by these dikes, which lead to mineralogy transformations, hydrothermal alteration and formation of hydraulic breccias (Fig. 2).

The stratigraphy of the Corumbataí Formation presented in Figure 2, according to Zanardo et al. (2016), can be divided into five mineralogical-textural levels that are composed predominantly by clayey siltstones interbedded with sandy siltstones, consisting of illite, quartz, feldspars, carbonates, hematite, montmorillonite, chlorite and zeolites, of authigenic and/or detrital origin, within a maximum thickness of $130 \mathrm{~m}$.

P1 mine corresponds to the bottom portion of Corumbataí Formation and it is characterized by plastic material in high amounts throughout the mine, even in the basal strata. Although P2 mine is close to P1 mine, it has different characteristics since it is located within the intermediate to upper portions of the Corumbataí Formation stratigraphic column, in which there are high amounts of carbonates in some of its layers.

P3 and P4 mines begin in the bottom portion of Irati Formation and extent to the intermediate portion of Corumbataí Formation, both with intense weathered claystones in their upper portions. P5 mine correlates to the upper portion of $\mathrm{P} 3$ and $\mathrm{P} 4$ mines.

P6, P7 and P8 mines are positioned in the bottom portion of the Corumbataí Formation, however they cover a much less portion of the stratigraphic column, since they do not present the upper portion seen in P3 and P4 mines. Also, in these mines clays there is more plastic and they present pronounced weathering alteration characteristics in the upper layers, next to the contact with Rio Claro Formation.

P9 mine has four distinct strata, with only the middle layer of the profile representing the extracted volume for

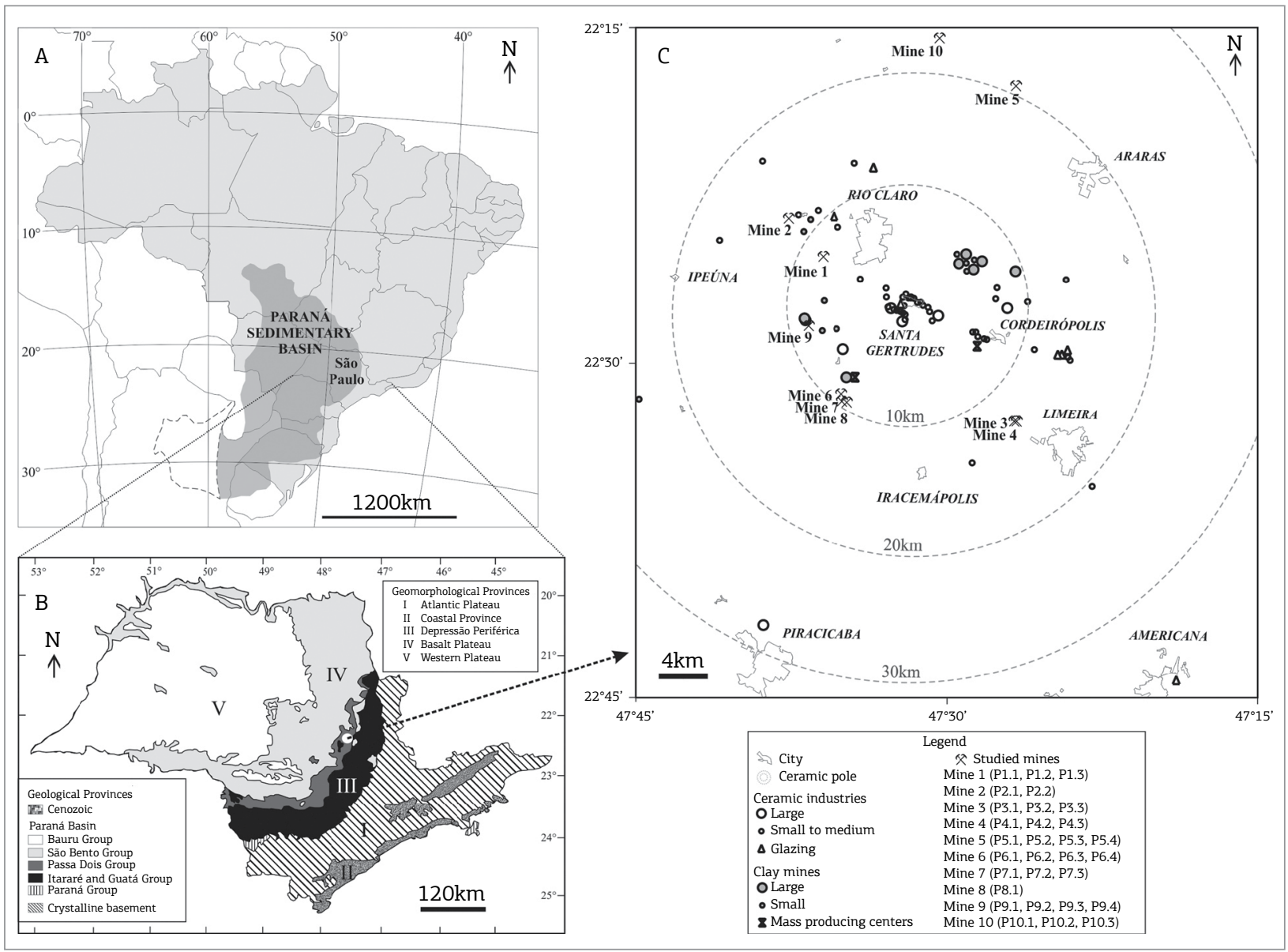

Figure 1. Location of the Paraná Basin in South America (A), simplified map with general geological and geomorphological features in São Paulo State (B) and the sampling site location within SGCP (C). Modified: Zálan et al. (1990), Penteado (1976) and Costa (2006). 


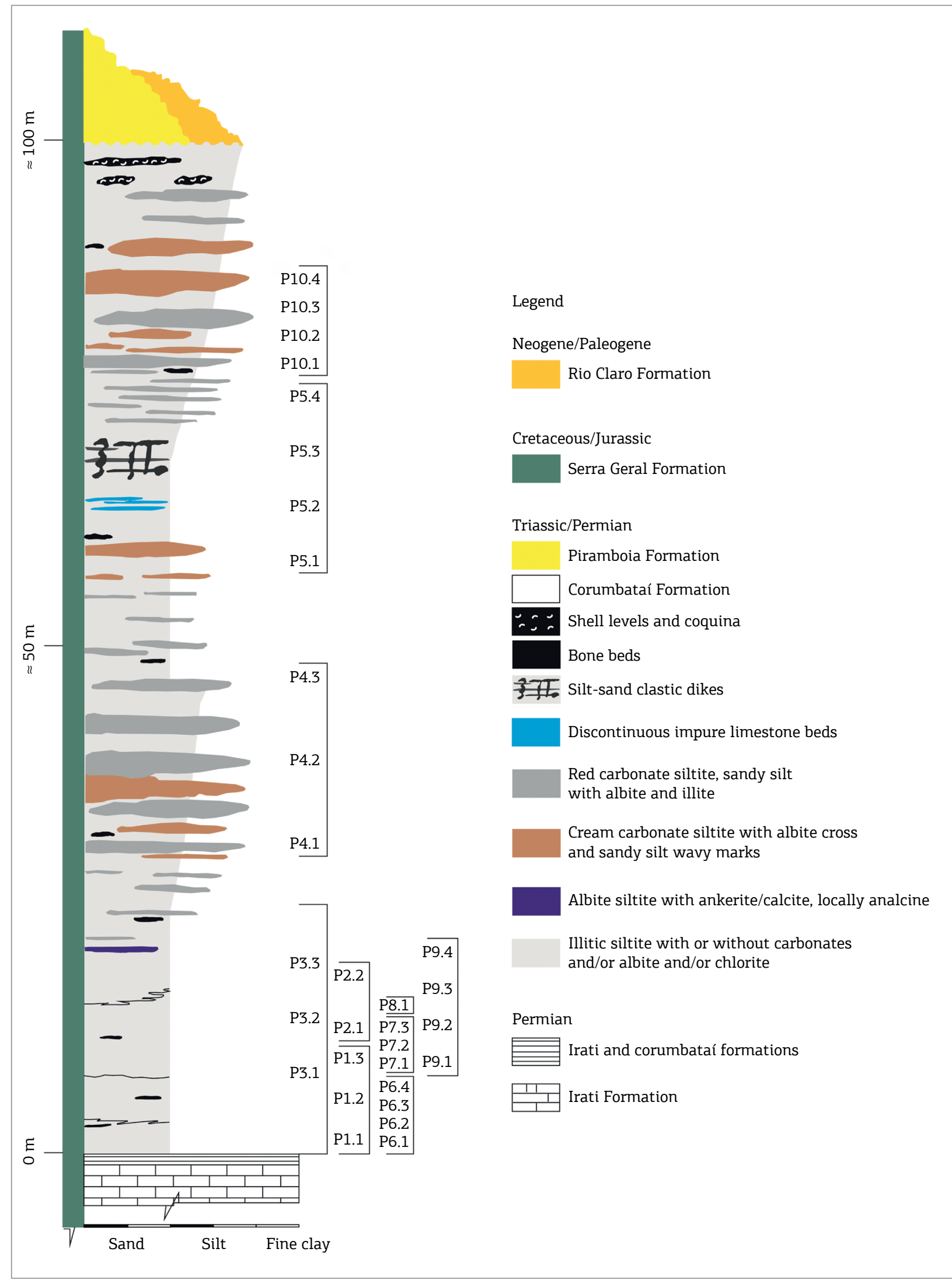

Figure 2. Schematic representation of the Corumbataí Formation in the SGCP region, with studied mines and its stratigraphic position. Modified: Zanardo et al. (2016). Mine 1 (P1.1, P1.2, P1.3); Mine 2 (P2.1, P2.2); Mine 3 (P3.1, P3.2, P3.3); Mine 4 (P4.1, P4.2, P4.3); Mine 5 (P5.1, P5.2, P5.3, P5.4); Mine 6 (P6.1, P6.2, P6.3, P6.4); Mine 7 (P7.1, P7.2, P7.3); Mine 8 (P8.1); Mine 9 (P9.1, P9.2, P9.3, P9.4) and Mine 10 (P10.1, P10.2, P10.3). 
ceramic masses, the upper weathered and plastic clayish material being discarded and the bottom portion materials used in low percentage.

P10 mine is considered an especial occurrence among the studied mines since it is characterized by prominent amounts of carbonates, partially resembling P2 mine, and being situated in the upper portion of the Corumbataí Formation stratigraphic column.

\section{MINERALOGY}

Illite, quartz and feldspar (albite and/or microcline) are the main minerals (Fig. 3) and three groups of samples were identified according to their mineralogy. The first group (G1) has the highest calcite content and low amounts of microcline. In the second group (G2), the quantity of calcite decreases and a marked content of albite appears. The third group (G3) is marked by the presence of kaolinite, a feature that distinguishes this group from the other two ones.
The decrease in calcite amounts towards upper samples can be attributed to weathering effects that causes its disintegration. The highest amounts of albite in G2 interfere with microcline reflections by hiding them, but their presence cannot be discarded since they reappear in the upper samples, most likely because weathering effects disintegrates albite, which allows the observation of microcline reflections. The increased amounts of kaolinite towards the upper samples of the profiles possibly indicate that they were neoformed, therefore reflecting a more advanced weathering state of these samples.

Chlorite occurs in samples from the bottom portion of the profiles (Fig. 3; samples in G1; P3.1, and P4.1, in G2) as indicative of the intense reflections compared to the upper samples (Fig. 3; P5; P6; P7; P9; all in G2). The amounts of chlorite in upper portion samples can be attributed to the weathering reactions that intensely affected the surface samples.

The weathering effect in quartz and albite is less evident among the samples of each profile since they are present in similar amounts along the profiles. Only in G3, the

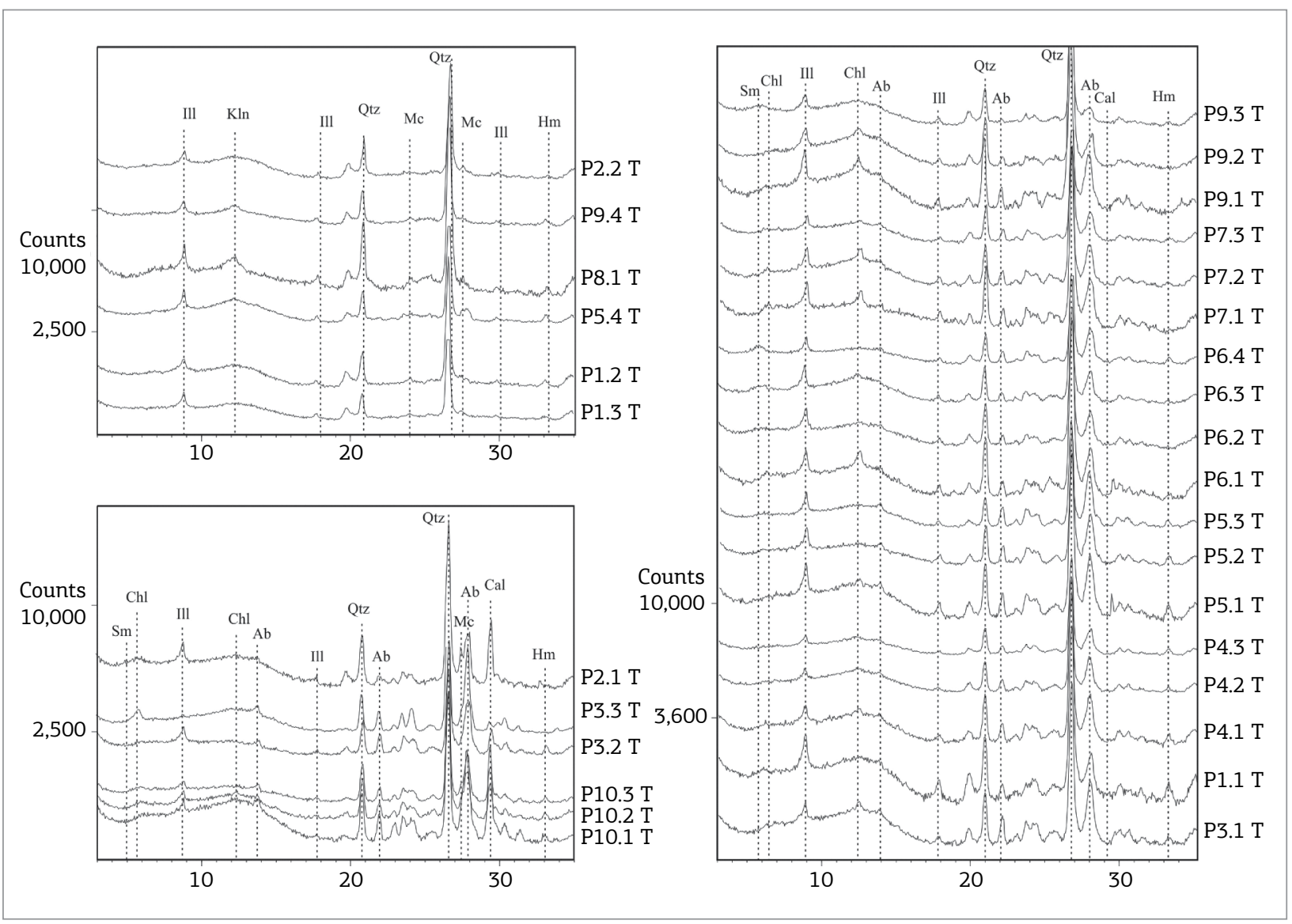

Figure 3. Diffractograms for each mine profile. Where: T, total sample; Ill, illite; Kln, kaolinite; Sm, smectite; Chl, chlorite; Qtz, quartz; Mc, microcline; Ab, albite; Cal, calcite; Hm, hematite. 
reflections of albite show a decrease in intensity, which makes the reflections of microcline visible (Fig. 3).

Mines 6 and 7 show similar mineralogy, composed by illite, smectite, chlorite, quartz and albite, and, although Mine 8 is very close to these mines in space, it presents kaolinite and absence of smectite/chlorite in the upper level of the profile, which indicates that Mine 8 is more weathered than mines 6 and 7.

In Mine 10 the samples present a particular mineralogy, with calcite being the main mineral, noted by the increased amounts and well-defined reflections in Fig. 3 (G1).

\section{WHOLE-ROCK GEOCHEMISTRY}

The results of major and trace elements analyses for the studied samples are listed in Table 1, as well as the compositions of the Upper Crust (UC, Taylor \& McLennan 1985), Average Post-Archean Australian Shale (PAAS, Taylor \& McLennan 1985) and Average North American Shale Composite (NASC, Gromet et al. 1984, Taylor \& McLennan 1985) for comparison.

The $\mathrm{SiO}_{2}$ content varies from 55.3 to $70.3 \%$, with the average value of $64.8 \%$, among the studied mines. Along each

Table 1. Chemical composition of major elements as wt\% and trace elements in ppm. Total $\mathrm{Fe}$ as $\mathrm{Fe}_{2} \mathrm{O}_{3}$, major and minor elements in \% and trace elements in ppm, UC (data from Taylor \& McLennan 1985), PAAS (data from Taylor \& McLennan 1985) and NASC (data from Gromet et al. 1984 and Taylor \& McLennan 1985).

\begin{tabular}{|c|c|c|c|c|c|c|c|c|c|c|c|}
\hline \multirow{2}{*}{ Sample } & \multicolumn{3}{|c|}{ Mine 1} & \multicolumn{2}{|c|}{ Mine 2} & \multicolumn{3}{|c|}{ Mine 3} & \multicolumn{3}{|c|}{ Mine 4} \\
\hline & P1.1 & P1.2 & P1.3 & P2.1 & P2.2 & P3.1 & P3.2 & P3.3 & P4.1 & P4.2 & P4. 3 \\
\hline $\mathrm{SiO}_{2}$ & 65.75 & 63.07 & 62.10 & 61.34 & 60.11 & 66.96 & 63.22 & 68.70 & 66.85 & 66.63 & 65.45 \\
\hline $\mathrm{Al}_{2} \mathrm{O}_{3}$ & 14.76 & 15.65 & 15.58 & 11.30 & 16.17 & 13.92 & 13.48 & 12.39 & 13.77 & 14.19 & 15.09 \\
\hline $\mathrm{Fe}_{2} \mathrm{O}_{3}$ & 5.86 & 5.55 & 5.47 & 3.95 & 5.61 & 5.30 & 4.82 & 3.07 & 5.45 & 5.35 & 5.59 \\
\hline $\mathrm{MgO}$ & 1.97 & 2.16 & 2.35 & 2.06 & 2.38 & 2.01 & 2.39 & 2.17 & 2.03 & 1.91 & 1.74 \\
\hline $\mathrm{CaO}$ & 0.60 & 0.28 & 0.28 & 5.84 & 0.27 & 1.01 & 3.04 & 1.95 & 0.73 & 0.62 & 0.62 \\
\hline $\mathrm{K}_{2} \mathrm{O}$ & 3.59 & 3.67 & 3.56 & 2.70 & 3.44 & 3.47 & 3.17 & 1.62 & 3.63 & 3.54 & 3.40 \\
\hline $\mathrm{Na}_{2} \mathrm{O}$ & 1.14 & 0.14 & 0.08 & 1.39 & 0.10 & 2.98 & 3.52 & 4.82 & 2.40 & 2.42 & 2.34 \\
\hline $\mathrm{MnO}$ & 0.04 & 0.05 & 0.05 & 0.11 & 0.06 & 0.06 & 0.10 & 0.07 & 0.04 & 0.05 & 0.09 \\
\hline $\mathrm{TiO}_{2}$ & 0.62 & 0.63 & 0.65 & 0.45 & 0.66 & 0.60 & 0.57 & 0.49 & 0.58 & 0.61 & 0.65 \\
\hline $\mathrm{P}_{2} \mathrm{O}_{5}$ & 0.18 & 0.07 & 0.07 & 0.15 & 0.10 & 0.24 & 0.14 & 0.16 & 0.26 & 0.17 & 0.16 \\
\hline LOI & 5.3 & 8.5 & 9.6 & 10.4 & 10.9 & 3.2 & 5.3 & 4.4 & 4.1 & 4.3 & 4.6 \\
\hline Sum & 99.81 & 99.77 & 99.79 & 99.69 & 99.80 & 99.75 & 99.75 & 99.84 & 99.84 & 99.79 & 99.73 \\
\hline Sc & 13.0 & 13.0 & 13.0 & 11.0 & 13.0 & 13.0 & 11.0 & 9.0 & 13.0 & 13.0 & 13.0 \\
\hline $\mathrm{Cr}$ & 0.01 & 0.01 & 0.01 & 0.01 & 0.01 & 0.01 & 0.02 & 0.00 & 0.01 & 0.01 & 0.02 \\
\hline $\mathrm{Ni}$ & 26.0 & 20.0 & 39.0 & 22.0 & 25.0 & 29.0 & 27.0 & 20.0 & 32.0 & 32.0 & 34.0 \\
\hline As & 4.4 & 2.7 & 2.0 & 5.3 & 3.5 & 6.1 & 10.0 & 15.0 & 1.9 & 2.2 & 6.4 \\
\hline $\mathrm{Rb}$ & 179 & 234 & 220 & 130 & 203 & 207 & 171 & 59 & 203 & 204 & 191 \\
\hline $\mathrm{Sr}$ & 179 & 92 & 86 & 297 & 79 & 205 & 194 & 130 & 181 & 160 & 128 \\
\hline $\mathrm{Zr}$ & 134 & 163 & 164 & 128 & 147 & 146 & 149 & 206 & 137 & 140 & 173 \\
\hline Cs & 8 & 11 & 13 & 8 & 12 & 10 & 10 & 2 & 8 & 9 & 10 \\
\hline $\mathrm{Ba}$ & 473 & 752 & 642 & 1451 & 576 & 491 & 488 & 579 & 412 & 464 & 554 \\
\hline $\mathrm{La}$ & 31.2 & 36.4 & 44.9 & 28.0 & 61.7 & 31.6 & 28.6 & 32.6 & 28.5 & 30.7 & 39.9 \\
\hline $\mathrm{Ce}$ & 65.1 & 74.1 & 89.0 & 58.7 & 110.4 & 65.5 & 62.8 & 68.9 & 58.0 & 64.0 & 79.6 \\
\hline $\operatorname{Pr}$ & 7.48 & 9.59 & 12.62 & 6.67 & 14.85 & 7.47 & 7.09 & 7.83 & 6.77 & 7.29 & 9.84 \\
\hline $\mathrm{Nd}$ & 28.3 & 39.7 & 53.8 & 25.7 & 55.6 & 27.2 & 27.5 & 30.0 & 25.3 & 27.3 & 36.9 \\
\hline $\mathrm{Sm}$ & 5.57 & 6.97 & 9.83 & 4.40 & 9.45 & 5.12 & 4.94 & 5.22 & 4.89 & 5.03 & 7.06 \\
\hline $\mathrm{Eu}$ & 1.03 & 1.42 & 1.92 & 0.84 & 1.83 & 0.94 & 0.91 & 1.05 & 0.94 & 1.00 & 1.41 \\
\hline $\mathrm{Gd}$ & 4.79 & 6.34 & 8.46 & 3.66 & 7.21 & 4.20 & 4.11 & 4.32 & 3.88 & 4.14 & 6.16 \\
\hline $\mathrm{Tb}$ & 0.77 & 0.96 & 1.37 & 0.61 & 1.16 & 0.73 & 0.69 & 0.74 & 0.70 & 0.71 & 1.01 \\
\hline
\end{tabular}


Letícia Hirata Godoy et al.

Table 1. Continuation.

\begin{tabular}{|c|c|c|c|c|c|c|c|c|c|c|c|}
\hline \multirow{2}{*}{ Sample } & \multicolumn{3}{|c|}{ Mine 1} & \multicolumn{2}{|c|}{ Mine 2} & \multicolumn{3}{|c|}{ Mine 3} & \multicolumn{3}{|c|}{ Mine 4} \\
\hline & P1.1 & P1.2 & P1.3 & P2.1 & P2.2 & P3.1 & P3.2 & P3.3 & P4.1 & P4.2 & P4.3 \\
\hline Dy & 4.24 & 5.06 & 7.38 & 3.47 & 6.57 & 4.01 & 3.88 & 4.00 & 3.87 & 3.67 & 5.40 \\
\hline Ho & 0.86 & 1.02 & 1.39 & 0.66 & 1.17 & 0.84 & 0.74 & 0.84 & 0.77 & 0.75 & 1.10 \\
\hline Er & 2.49 & 2.77 & 3.87 & 2.00 & 3.30 & 2.42 & 2.37 & 2.35 & 2.27 & 2.19 & 3.19 \\
\hline $\mathrm{Tm}$ & 0.39 & 0.43 & 0.59 & 0.31 & 0.52 & 0.37 & 0.35 & 0.36 & 0.35 & 0.36 & 0.47 \\
\hline $\mathrm{Yb}$ & 2.42 & 2.84 & 3.66 & 1.78 & 3.01 & 2.39 & 2.17 & 2.41 & 2.25 & 2.06 & 3.01 \\
\hline $\mathrm{Lu}$ & 0.37 & 0.40 & 0.58 & 0.31 & 0.50 & 0.37 & 0.33 & 0.37 & 0.36 & 0.33 & 0.46 \\
\hline $\mathrm{Hf}$ & 4.40 & 4.80 & 5.10 & 4.40 & 4.60 & 4.30 & 4.60 & 6.30 & 4.30 & 4.20 & 5.40 \\
\hline $\mathrm{Ta}$ & 1.00 & 1.10 & 1.10 & 0.70 & 1.10 & 1.00 & 0.90 & 0.90 & 0.90 & 0.90 & 1.20 \\
\hline W & 2.20 & 1.70 & 2.00 & 1.60 & 1.70 & 3.30 & 1.90 & 2.50 & 1.80 & 1.80 & 2.40 \\
\hline Th & 14.10 & 14.90 & 16.30 & 9.50 & 17.80 & 13.90 & 14.00 & 12.00 & 11.80 & 12.30 & 13.20 \\
\hline $\mathrm{U}$ & 2.50 & 2.40 & 2.30 & 4.40 & 3.50 & 2.10 & 2.30 & 2.70 & 2.10 & 2.20 & 2.30 \\
\hline \multirow{2}{*}{ Sample } & \multicolumn{4}{|c|}{ Mine 5} & \multicolumn{4}{|c|}{ Mine 6} & \multicolumn{3}{|c|}{ Mine 7} \\
\hline & P5.1 & P5.2 & P5.3 & P5.4 & P6.1 & P6.2 & P6.3 & P6.4 & P7.1 & P7.2 & P7.3 \\
\hline $\mathrm{SiO}_{2}$ & 67.66 & 67.76 & 66.72 & 61.42 & 68.25 & 65.62 & 65.85 & 65.59 & 68.79 & 67.14 & 66.85 \\
\hline $\mathrm{Al}_{2} \mathrm{O}_{3}$ & 14.18 & 14.08 & 14.73 & 17.63 & 13.06 & 14.20 & 14.09 & 13.77 & 13.20 & 14.58 & 13.68 \\
\hline $\mathrm{Fe}_{2} \mathrm{O}_{3}$ & 4.77 & 4.66 & 4.26 & 6.22 & 5.13 & 5.12 & 5.30 & 4.73 & 4.97 & 5.14 & 5.02 \\
\hline $\mathrm{MgO}$ & 1.70 & 1.82 & 1.95 & 1.58 & 2.28 & 2.28 & 2.38 & 2.28 & 2.19 & 2.09 & 1.79 \\
\hline $\mathrm{CaO}$ & 0.58 & 0.75 & 0.84 & 0.15 & 0.96 & 0.54 & 0.64 & 1.04 & 0.61 & 0.48 & 1.10 \\
\hline $\mathrm{K}_{2} \mathrm{O}$ & 3.64 & 3.48 & 3.07 & 2.95 & 3.36 & 3.40 & 3.42 & 3.13 & 3.31 & 3.58 & 3.29 \\
\hline $\mathrm{Na}_{2} \mathrm{O}$ & 2.63 & 3.02 & 3.78 & 0.85 & 1.74 & 1.80 & 1.99 & 2.63 & 2.11 & 2.54 & 2.14 \\
\hline $\mathrm{MnO}$ & 0.02 & 0.04 & 0.05 & 0.07 & 0.03 & 0.03 & 0.03 & 0.06 & 0.03 & 0.03 & 0.04 \\
\hline $\mathrm{TiO}_{2}$ & 0.61 & 0.59 & 0.57 & 0.74 & 0.56 & 0.60 & 0.60 & 0.57 & 0.58 & 0.62 & 0.57 \\
\hline $\mathrm{P}_{2} \mathrm{O}_{5}$ & 0.13 & 0.14 & 0.16 & 0.06 & 0.34 & 0.12 & 0.14 & 0.14 & 0.17 & 0.11 & 0.57 \\
\hline LOI & 3.9 & 3.5 & 3.7 & 8.1 & 4.1 & 6.1 & 5.4 & 5.9 & 3.9 & 3.5 & 4.8 \\
\hline Sum & 99.82 & 99.84 & 99.83 & 99.77 & 99.81 & 99.81 & 99.84 & 99.84 & 99.86 & 99.81 & 99.85 \\
\hline Sc & 12.0 & 11.0 & 11.0 & 15.0 & 13.0 & 12.0 & 12.0 & 11.0 & 12.0 & 11.0 & 12.0 \\
\hline $\mathrm{Cr}$ & 0.01 & 0.01 & 0.01 & 0.02 & 0.01 & 0.01 & 0.01 & 0.01 & 0.01 & 0.01 & 0.01 \\
\hline $\mathrm{Ni}$ & 28.0 & 62.0 & 20.0 & 25.0 & 26.0 & 43.0 & 57.0 & 25.0 & 32.0 & 22.0 & 22.0 \\
\hline As & 4.7 & 9.4 & 10.8 & 2.3 & 4.1 & 0.7 & 0.8 & 4.7 & 4.0 & 0.7 & 1.8 \\
\hline $\mathrm{Rb}$ & 202 & 192 & 153 & 174 & 172 & 173 & 173 & 156 & 188 & 210 & 214 \\
\hline $\mathrm{Sr}$ & 205 & 228 & 213 & 92 & 207 & 138 & 156 & 177 & 220 & 179 & 232 \\
\hline $\mathrm{Zr}$ & 158 & 181 & 166 & 202 & 163 & 136 & 141 & 161 & 154 & 144 & 166 \\
\hline Cs & 10 & 10 & 8 & 12 & 6 & 8 & 7 & 8 & 7 & 7 & 7 \\
\hline $\mathrm{Ba}$ & 489 & 521 & 639 & 661 & 467 & 526 & 475 & 531 & 519 & 478 & 552 \\
\hline $\mathrm{La}$ & 30.8 & 31.0 & 35.3 & 50.6 & 30.8 & 30.6 & 30.0 & 29.1 & 29.7 & 33.5 & 43.3 \\
\hline $\mathrm{Ce}$ & 66.4 & 65.8 & 75.1 & 92.3 & 67.4 & 66.9 & 65.5 & 62.7 & 63.8 & 70.2 & 84.7 \\
\hline $\operatorname{Pr}$ & 6.96 & 7.09 & 8.42 & 13.17 & 7.88 & 7.18 & 6.96 & 6.64 & 7.27 & 7.67 & 10.91 \\
\hline $\mathrm{Nd}$ & 26.6 & 25.8 & 33.2 & 51.6 & 30.3 & 27.3 & 27.6 & 23.4 & 28.6 & 28.5 & 43.4 \\
\hline $\mathrm{Sm}$ & 4.78 & 4.83 & 6.08 & 9.85 & 6.21 & 5.03 & 4.93 & 4.64 & 5.21 & 4.96 & 8.40 \\
\hline $\mathrm{Eu}$ & 0.89 & 0.90 & 1.06 & 2.10 & 1.15 & 0.91 & 0.88 & 0.83 & 0.97 & 0.91 & 1.55 \\
\hline $\mathrm{Gd}$ & 3.76 & 3.81 & 4.84 & 8.43 & 5.54 & 4.15 & 3.84 & 3.81 & 4.56 & 4.04 & 7.77 \\
\hline $\mathrm{Tb}$ & 0.67 & 0.66 & 0.83 & 1.36 & 0.92 & 0.68 & 0.66 & 0.66 & 0.73 & 0.68 & 1.22 \\
\hline
\end{tabular}


Table 1. Continuation.

\begin{tabular}{|c|c|c|c|c|c|c|c|c|c|c|c|}
\hline \multirow{2}{*}{ Sample } & \multicolumn{4}{|c|}{ Mine 5} & \multicolumn{4}{|c|}{ Mine 6} & \multicolumn{3}{|c|}{ Mine 7} \\
\hline & P5.1 & P5.2 & P5.3 & P5.4 & P6.1 & P6.2 & P6.3 & P6.4 & P7.1 & P7.2 & P7.3 \\
\hline Dy & 3.52 & 3.64 & 4.39 & 7.08 & 4.75 & 3.64 & 3.57 & 3.70 & 4.09 & 3.70 & 6.80 \\
\hline Ho & 0.74 & 0.76 & 0.88 & 1.41 & 0.93 & 0.79 & 0.69 & 0.75 & 0.85 & 0.74 & 1.35 \\
\hline Er & 2.31 & 2.23 & 2.65 & 3.93 & 2.67 & 2.20 & 2.27 & 2.29 & 2.38 & 2.25 & 3.73 \\
\hline $\mathrm{Tm}$ & 0.34 & 0.35 & 0.42 & 0.61 & 0.40 & 0.33 & 0.33 & 0.35 & 0.37 & 0.37 & 0.56 \\
\hline $\mathrm{Yb}$ & 2.45 & 2.25 & 2.39 & 3.82 & 2.57 & 2.16 & 2.17 & 2.11 & 2.50 & 2.28 & 3.39 \\
\hline $\mathrm{Lu}$ & 0.36 & 0.36 & 0.38 & 0.57 & 0.37 & 0.33 & 0.33 & 0.34 & 0.37 & 0.34 & 0.50 \\
\hline $\mathrm{Hf}$ & 4.50 & 5.50 & 4.50 & 6.00 & 4.80 & 4.00 & 4.20 & 4.40 & 4.30 & 4.50 & 4.40 \\
\hline $\mathrm{Ta}$ & 0.90 & 1.00 & 1.10 & 1.30 & 0.90 & 1.10 & 1.00 & 1.00 & 0.90 & 1.10 & 1.00 \\
\hline W & 1.50 & 1.70 & 2.50 & 3.30 & 2.30 & 1.80 & 2.20 & 1.90 & 2.80 & 1.80 & 2.10 \\
\hline Th & 12.90 & 13.40 & 17.90 & 15.70 & 11.80 & 14.10 & 13.50 & 12.10 & 14.30 & 14.30 & 14.20 \\
\hline $\mathrm{U}$ & 2.90 & 2.70 & 2.80 & 3.60 & 2.30 & 2.40 & 2.50 & 2.30 & 2.30 & 2.30 & 3.00 \\
\hline \multirow{2}{*}{ Sample } & Mine 8 & \multicolumn{4}{|c|}{ Mine 9} & \multicolumn{3}{|c|}{ Mine 10} & \multirow{2}{*}{ UC } & \multirow{2}{*}{ NASC } & \multirow{2}{*}{ PAAS } \\
\hline & P8.1 & P9.1 & P9.2 & P9.3 & P9.4 & P10.1 & P10.2 & P10.3 & & & \\
\hline $\mathrm{SiO}_{2}$ & 67.44 & 70.32 & 68.16 & 63.15 & 63.51 & 55.32 & 61.34 & 63.30 & 66.00 & 64.80 & 62.80 \\
\hline $\mathrm{Al}_{2} \mathrm{O}_{3}$ & 15.19 & 12.83 & 13.51 & 14.71 & 15.89 & 9.74 & 12.27 & 11.45 & 15.20 & 16.90 & 18.90 \\
\hline $\mathrm{Fe}_{2} \mathrm{O}_{3}$ & 5.69 & 4.99 & 5.05 & 5.64 & 6.78 & 2.96 & 4.39 & 3.79 & 5.00 & 5.65 & 7.22 \\
\hline $\mathrm{MgO}$ & 1.14 & 2.18 & 2.17 & 1.99 & 1.53 & 1.77 & 3.28 & 2.13 & 2.20 & 2.86 & 2.20 \\
\hline $\mathrm{CaO}$ & 0.05 & 0.67 & 0.79 & 0.41 & 0.16 & 12.63 & 4.78 & 5.52 & 4.20 & 3.63 & 1.30 \\
\hline $\mathrm{K}_{2} \mathrm{O}$ & 2.28 & 3.11 & 3.34 & 3.73 & 3.08 & 1.86 & 3.31 & 2.27 & 3.40 & 3.97 & 3.70 \\
\hline $\mathrm{Na}_{2} \mathrm{O}$ & 0.09 & 2.15 & 1.48 & 0.83 & 0.09 & 3.19 & 2.74 & 2.92 & 3.90 & 1.14 & 1.20 \\
\hline $\mathrm{MnO}$ & 0.03 & 0.03 & 0.04 & 0.06 & 0.06 & 0.15 & 0.08 & 0.08 & 0.08 & 0.06 & 0.11 \\
\hline $\mathrm{TiO}_{2}$ & 0.65 & 0.57 & 0.58 & 0.63 & 0.67 & 0.39 & 0.51 & 0.47 & 0.50 & 0.70 & 1.00 \\
\hline $\mathrm{P}_{2} \mathrm{O}_{5}$ & 0.10 & 0.09 & 0.34 & 0.08 & 0.07 & 0.14 & 0.21 & 0.13 & - & 0.13 & 0.16 \\
\hline LOI & 7.2 & 2.9 & 4.4 & 8.6 & 8.0 & 11.7 & 6.9 & 7.8 & - & - & 6.00 \\
\hline Sum & 99.86 & 99.84 & 99.86 & 99.83 & 99.84 & 99.85 & 99.81 & 99.86 & 99.84 & 100.48 & 104.59 \\
\hline Sc & 14.0 & 11.0 & 11.0 & 13.0 & 14.0 & 9.0 & 10.0 & 8.0 & 10.0 & 14.9 & 16.0 \\
\hline $\mathrm{Cr}$ & 0.01 & 0.01 & 0.01 & 0.01 & 0.01 & 0.00 & 0.01 & 0.01 & 35 & 125 & 110 \\
\hline $\mathrm{Ni}$ & 20.0 & 20.0 & 24.0 & 30.0 & 35.0 & 20.0 & 20.0 & 20.0 & 20.0 & 58.0 & 55.0 \\
\hline As & 2.1 & 6.3 & 1.5 & 3.3 & 0.5 & 3.7 & 14.9 & 9.6 & & 28.4 & - \\
\hline $\mathrm{Rb}$ & 185 & 169 & 191 & 231 & 231 & 91 & 166 & 96 & 110 & 125 & 160 \\
\hline $\mathrm{Sr}$ & 60 & 215 & 241 & 147 & 118 & 323 & 274 & 230 & 350 & 142 & 200 \\
\hline $\mathrm{Zr}$ & 161 & 146 & 151 & 149 & 160 & 161 & 152 & 176 & 240 & 200 & 210 \\
\hline Cs & 7 & 7 & 8 & 9 & 9 & 5 & 8 & 5 & 4 & 5 & 15 \\
\hline $\mathrm{Ba}$ & 353 & 472 & 504 & 611 & 535 & 602 & 588 & 655 & 700 & 636 & 650 \\
\hline $\mathrm{La}$ & 47.8 & 29.6 & 32.7 & 35.4 & 40.4 & 31.1 & 29.0 & 30.0 & 30.0 & 31.1 & 38.2 \\
\hline $\mathrm{Ce}$ & 98.5 & 64.3 & 67.9 & 71.7 & 79.9 & 62.4 & 61.5 & 60.5 & 64.0 & 67.033 & 79.6 \\
\hline $\operatorname{Pr}$ & 11.15 & 6.97 & 8.09 & 8.23 & 10.10 & 7.23 & 7.16 & 7.16 & 7.10 & & 8.83 \\
\hline $\mathrm{Nd}$ & 42.8 & 27.3 & 32.6 & 31.6 & 41.0 & 27.2 & 28.1 & 27.9 & 26.0 & 30.4 & 33.9 \\
\hline $\mathrm{Sm}$ & 6.75 & 4.88 & 6.21 & 5.49 & 8.42 & 4.92 & 5.13 & 5.25 & 4.50 & 5.98 & 5.55 \\
\hline $\mathrm{Eu}$ & 1.20 & 0.89 & 1.14 & 0.98 & 1.79 & 0.93 & 0.98 & 1.03 & 0.88 & 1.253 & 1.08 \\
\hline $\mathrm{Gd}$ & 5.27 & 4.23 & 5.52 & 4.71 & 11.12 & 4.21 & 4.43 & 5.03 & 3.80 & 5.5 & 4.66 \\
\hline $\mathrm{Tb}$ & 0.82 & 0.67 & 0.89 & 0.73 & 1.90 & 0.71 & 0.69 & 0.83 & 0.64 & 0.85 & 0.77 \\
\hline
\end{tabular}


Table 1. Continuation.

\begin{tabular}{|c|c|c|c|c|c|c|c|c|c|c|c|}
\hline \multirow{2}{*}{ Sample } & \multirow{2}{*}{$\begin{array}{c}\text { Mine } 8 \\
\text { P8.1 }\end{array}$} & \multicolumn{4}{|c|}{ Mine 9} & \multicolumn{3}{|c|}{ Mine 10} & \multirow{2}{*}{ UC } & \multirow{2}{*}{ NASC } & \multirow{2}{*}{ PAAS } \\
\hline & & P9.1 & P9.2 & P9.3 & P9.4 & P10.1 & P10.2 & P10.3 & & & \\
\hline Dy & 4.77 & 3.77 & 5.06 & 4.33 & 12.28 & 4.01 & 3.69 & 4.68 & 3.50 & 5.54 & 4.68 \\
\hline Ho & 0.92 & 0.75 & 0.99 & 0.85 & 2.77 & 0.81 & 0.78 & 1.04 & 0.80 & & 0.99 \\
\hline $\mathrm{Er}$ & 2.63 & 2.15 & 2.84 & 2.48 & 7.61 & 2.50 & 2.31 & 2.99 & 2.30 & 3.275 & 2.85 \\
\hline $\mathrm{Tm}$ & 0.42 & 0.34 & 0.44 & 0.40 & 1.09 & 0.39 & 0.35 & 0.44 & 0.33 & & 0.41 \\
\hline $\mathrm{Yb}$ & 2.74 & 2.28 & 2.72 & 2.53 & 6.41 & 2.81 & 2.24 & 2.81 & 2.20 & 3.113 & 2.82 \\
\hline $\mathrm{Lu}$ & 0.41 & 0.34 & 0.41 & 0.38 & 0.94 & 0.43 & 0.33 & 0.41 & 0.32 & 0.456 & 0.43 \\
\hline $\mathrm{Hf}$ & 4.80 & 4.30 & 4.40 & 4.60 & 4.60 & 4.70 & 4.70 & 5.10 & 5.8 & 6.3 & - \\
\hline $\mathrm{Ta}$ & 1.10 & 1.10 & 1.00 & 1.10 & 1.10 & 0.70 & 0.90 & 0.80 & - & 1.1 & 14.60 \\
\hline $\mathrm{W}$ & 2.00 & 4.90 & 2.00 & 2.10 & 2.20 & 2.30 & 2.40 & 2.20 & - & 2.1 & - \\
\hline Th & 14.10 & 13.80 & 13.30 & 15.60 & 16.00 & 11.30 & 11.80 & 11.60 & 10.5 & 12.3 & 3.10 \\
\hline $\mathrm{U}$ & 2.40 & 2.40 & 2.80 & 3.00 & 2.70 & 3.50 & 7.00 & 5.30 & 2.5 & 2.66 & - \\
\hline
\end{tabular}

UC: Upper Crust; PAAS: Average Post-Archaean Australian Shale; NASC: Average North American Shale Composite.

profile, these values slightly decrease from lower to upper levels. The $\mathrm{SiO}_{2}$ content in the samples is attributed mainly to the presence of clay minerals, plagioclase and quartz and it varies according to the evolution of the weathering hydrolyses reactions. The smallest contents of this oxide are found in the carbonated samples from Mine 10, in which the $\mathrm{CaO}$ content increases.

The $\mathrm{CaO}$ has the same behavior as $\mathrm{SiO}_{2}$ of lower values in the upper levels, 0.1 to $12.6 \%$ and the average of $1.8 \%$. The decreased content is consequence of the dissolution of calcite that occurs as an interstitial diagenetic mineral within the Corumbataí Formation claystones.

The $\mathrm{Al}_{2} \mathrm{O}_{3}$ has between 9.7 and $17.6 \%$ with the average of $13.8 \%$ among the mines. Along the profiles, the $\mathrm{Al}_{2} \mathrm{O}_{3}$ content presents an opposite behavior when compared to $\mathrm{SiO}_{2}$ in consequence of $\mathrm{Si}$ to be more mobile than $\mathrm{Al}_{2} \mathrm{O}_{3}$. $\mathrm{Fe}_{2} \mathrm{O}_{3}$ and $\mathrm{TiO}_{2}$ oxides and the loss on ignition (LOI) also increase upward and vary between 2.7 to $6.8 \%$ ( $4.9 \%$ average), 0.4 to $0.7 \%$ ( $0.6 \%$ average) and 2.9 to $13.7 \%$ (6.4 average), respectively.

The $\mathrm{MgO}$ and $\mathrm{K}_{2} \mathrm{O}$ content are practically constant towards the upper levels. The average concentrations are $2.2 \%$ (1.1 to $4.4 \%$ ) and $3.2 \%$ (1.6 to $3.8 \%$ ), respectively. The constant content of these two elements along the profiles is consequence of neoformation of smectite and illite that imprison $\mathrm{Mg}$ and $\mathrm{K}$ when dolomite was solubilized. The minor variation in the $\mathrm{MgO} / \mathrm{K}_{2} \mathrm{O}$ ratios when compared to $\mathrm{SiO}_{2}$ constitutes an excellent intrinsic feature that can be used for the selection of raw materials from the Corumbataí Formation for ceramic purposes, as previously pointed out by Moreno et al. (2014).

The $\mathrm{Na}_{2} \mathrm{O}, \mathrm{MnO}$ and $\mathrm{P}_{2} \mathrm{O}_{5}$ occur in minor proportions and vary along the profiles according to the presence of these elements in minor minerals such as plagioclase (albite), or as minor element substitutions, i.e., $\mathrm{Mn}$ in calcite and $\mathrm{P}$ in clay minerals.
The $\mathrm{Al}_{2} \mathrm{O}_{3}-\left(\mathrm{CaO}+\mathrm{Na}_{2} \mathrm{O}\right)-\mathrm{K}_{2} \mathrm{O}(\mathrm{A}-\mathrm{CN}-\mathrm{K})$ ternary diagram (Nesbitt \& Young, 1982, 1984, 1989) is used to access the weathering trends. The studied samples present a range of compositions that plot along the A-CN axes and away from the $\mathrm{K}$ axis (Fig. 4), suggesting different weathering histories. All samples present relative low contents in $\mathrm{K}_{2} \mathrm{O}$ due to the low content of K-feldspar, with K concentrated mainly within illite.

The chemical compositions of the samples for these oxides are similar to those ones of the UC, NASC and

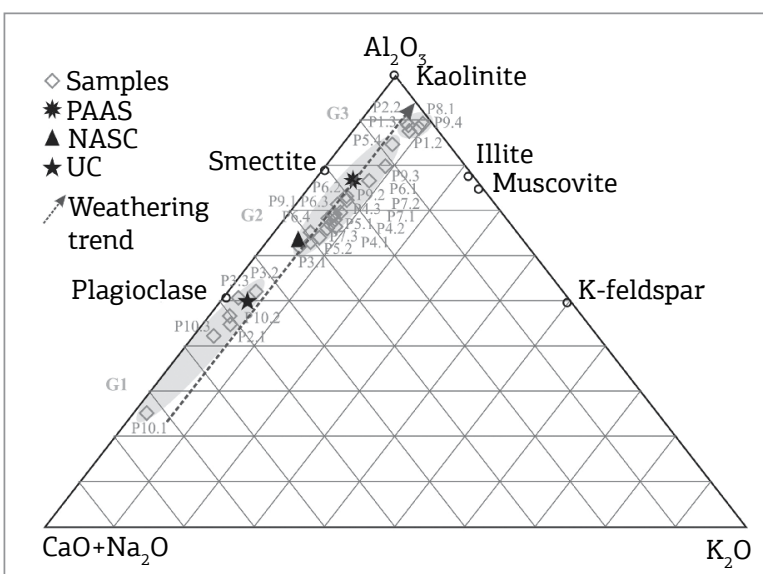

PAAS: Average Post-Archean Australian Shale; NASC: Average North American Shale Composite; UC: Upper Crust.

Figure 4. Ternary diagram of the $\left(\mathrm{Al}_{2} \mathrm{O}_{3}\right) /\left(\mathrm{CaO}+\mathrm{Na}_{2} \mathrm{O}\right) /$ $\left(\mathrm{K}_{2} \mathrm{O}\right)$ oxides, in molecular proportions, for the sedimentary samples (Nesbitt and Young, 1982, 1984 and 1989), in comparison to the Upper Crust (UC, data from Taylor and McLennan, 1985), Average PostArchaean Australian Shale (PAAS, data from Taylor and McLennan, 1985) and Average North American Shale Composite (N ASC, data from Gromet et al., 1984 and Taylor and McLennan, 1985). 
PAAS sediments, the first one as the least weathered representative and the last one as the most weathered one, respectively, according to the plotted data in Figure 4. Thus, the samples can be divided into three main groups, following the compositions of UC (G1), NASC and PAAS (G2) and one of an advanced weathering condition (G3), as shown in Figure 4.

The greater amounts of albite (Fig. 3), in (G1) indicate the immaturity of the sediments from the lower portion of Mine 2, Mine 3 and Mine 10. G2 corresponds to samples with intermediate (NASC composition) to relative advanced weathering state (PAAS composition).
The advanced weathering trend (G3) is marked by an increase in the relative contents of $\mathrm{Al}_{2} \mathrm{O}_{3}$, while the $\mathrm{CaO}$ and $\mathrm{Na}_{2} \mathrm{O}$ content decreases and is represented by the upper samples of Mines 1, 2, 8 and 9. In these samples, the relative increase in $\mathrm{Al}_{2} \mathrm{O}_{3}$ is in accordance to the neoformation of kaolinite, as seen in Fig. 3.

The chemical index of weathering (CIW) and chemical index of alteration (CIA) (Harnois 1988, Nesbitt \& Young, 1982) are calculated for all the samples and both show similar patterns (Tab. 2 and Fig. 5) that correlate to the observations made in the A-CN-K diagram. The highest values

Table 2. Chemical indexes calculated for the samples. $\mathrm{CIA}=\left[\mathrm{Al}_{2} \mathrm{O}_{3} /\left(\mathrm{Al}_{2} \mathrm{O}_{3}+\mathrm{CaO}+\mathrm{Na}_{2} \mathrm{O}+\mathrm{K}_{2} \mathrm{O}\right)\right] \times 100$ and $\mathrm{CIW}=\left[\mathrm{Al}_{2} \mathrm{O}_{3}\right.$ $\left./\left(\mathrm{Al}_{2} \mathrm{O}_{3}+\mathrm{CaO}+\mathrm{Na}_{2} \mathrm{O}\right)\right] \times 100$ in molecular proportions. $\mathrm{Eu} / \mathrm{Eu}^{*}=\mathrm{Eu}_{\mathrm{cn}} /\left[\left(\mathrm{Sm}_{\mathrm{cn}}\right)\left(\mathrm{Gd}_{\mathrm{cn}}\right)\right.$ and cn: chondrite normalized.

\begin{tabular}{|c|c|c|c|c|c|c|c|c|c|}
\hline Mines & Samples & CIA & CIW & $\mathrm{La} / \mathrm{Th}$ & $\mathrm{Th} / \mathrm{Sc}$ & $\mathbf{E u} / \mathbf{E u}^{*}$ & $\mathrm{La} / \mathrm{Lu} \mathrm{c}_{\mathrm{cn}}$ & $\mathrm{La} / \mathrm{Sm}_{\mathrm{cn}}$ & $\mathrm{Gd} / \mathrm{Lu} \mathbf{u}_{\mathrm{cr}}$ \\
\hline \multirow{3}{*}{ Mine 1} & P1.1 & 76.50 & 83.24 & 2.21 & 1.08 & 0.61 & 8.75 & 3.53 & 1.61 \\
\hline & P1.2 & 87.13 & 96.69 & 2.44 & 1.15 & 0.65 & 9.45 & 3.29 & 1.97 \\
\hline & P1.3 & 87.52 & 97.68 & 2.75 & 1.25 & 0.64 & 8.04 & 2.88 & 1.81 \\
\hline \multirow{2}{*}{ Mine 2} & P2.1 & 66.95 & 72.48 & 2.95 & 0.86 & 0.64 & 9.38 & 4.01 & 1.47 \\
\hline & P2.2 & 87.25 & 97.23 & 3.47 & 1.37 & 0.68 & 12.81 & 4.11 & 1.79 \\
\hline \multirow{3}{*}{ Mine 3} & P3.1 & 59.28 & 63.55 & 2.27 & 1.07 & 0.62 & 8.87 & 3.89 & 1.41 \\
\hline & P3.2 & 53.50 & 57.11 & 2.04 & 1.27 & 0.62 & 9.00 & 3.65 & 1.55 \\
\hline & P3.3 & 36.33 & 38.57 & 2.72 & 1.33 & 0.68 & 9.15 & 3.93 & 1.45 \\
\hline \multirow{3}{*}{ Mine 4} & P4.1 & 64.34 & 69.07 & 2.42 & 0.91 & 0.66 & 8.22 & 3.67 & 1.34 \\
\hline & P4.2 & 63.46 & 68.41 & 2.50 & 0.95 & 0.67 & 9.66 & 3.84 & 1.56 \\
\hline & P4.3 & 64.48 & 69.70 & 3.02 & 1.02 & 0.65 & 9.00 & 3.56 & 1.66 \\
\hline \multirow{4}{*}{ Mine 5} & P5.1 & 59.40 & 64.29 & 2.39 & 1.08 & 0.64 & 8.88 & 4.06 & 1.30 \\
\hline & P5.2 & 56.08 & 60.36 & 2.31 & 1.22 & 0.64 & 8.94 & 4.04 & 1.32 \\
\hline & P5.3 & 49.19 & 52.66 & 1.97 & 1.63 & 0.60 & 9.64 & 3.66 & 1.58 \\
\hline & P5.4 & 78.93 & 87.11 & 3.22 & 1.05 & 0.70 & 9.21 & 3.23 & 1.84 \\
\hline \multirow{4}{*}{ Mine 6} & P6.1 & 68.77 & 74.35 & 2.61 & 0.91 & 0.60 & 8.64 & 3.12 & 1.86 \\
\hline & P6.2 & 67.81 & 73.67 & 2.17 & 1.18 & 0.61 & 9.63 & 3.83 & 1.56 \\
\hline & P6.3 & 66.92 & 72.40 & 2.22 & 1.13 & 0.62 & 9.44 & 3.83 & 1.45 \\
\hline & P6.4 & 59.20 & 63.75 & 2.40 & 1.10 & 0.60 & 8.88 & 3.95 & 1.39 \\
\hline \multirow{3}{*}{ Mine 7} & P7.1 & 64.63 & 69.90 & 2.08 & 1.19 & 0.61 & 8.33 & 3.59 & 1.53 \\
\hline & P7.2 & 61.70 & 66.67 & 2.34 & 1.30 & 0.62 & 10.23 & 4.25 & 1.48 \\
\hline & P7.3 & 64.61 & 69.72 & 3.05 & 1.18 & 0.59 & 8.99 & 3.25 & 1.93 \\
\hline Mine 8 & P8.1 & 88.08 & 97.93 & 3.39 & 1.01 & 0.62 & 12.10 & 4.46 & 1.60 \\
\hline \multirow{4}{*}{ Mine 9} & P9.1 & 64.47 & 69.60 & 3.05 & 0.19 & 0.60 & 9.04 & 3.82 & 1.55 \\
\hline & P9.2 & 70.63 & 76.86 & 2.53 & 1.29 & 0.60 & 8.28 & 3.32 & 1.67 \\
\hline & P9.3 & 78.77 & 86.37 & 2.57 & 1.25 & 0.59 & 9.67 & 4.06 & 1.54 \\
\hline & P9.4 & 89.21 & 97.84 & 3.04 & 1.21 & 0.57 & 4.46 & 3.02 & 1.47 \\
\hline \multirow{3}{*}{ Mine 10} & P10.1 & 44.25 & 46.98 & 3.84 & 1.01 & 0.62 & 7.51 & 3.98 & 1.22 \\
\hline & P10.2 & 56.87 & 60.89 & 1.96 & 1.48 & 0.63 & 9.12 & 3.56 & 1.67 \\
\hline & P10.3 & 52.20 & 55.82 & 2.65 & 1.26 & 0.61 & 7.60 & 3.60 & 1.52 \\
\hline \multicolumn{2}{|c|}{ UC } & - & - & 2.62 & 0.91 & 0.65 & 9.73 & 4.20 & 1.48 \\
\hline \multicolumn{2}{|c|}{ NASC } & - & - & 2.80 & 1.07 & 0.67 & - & - & 1.50 \\
\hline \multicolumn{2}{|c|}{ PAAS } & - & - & 2.53 & 0.83 & 0.65 & 9.16 & 4.33 & 1.34 \\
\hline
\end{tabular}

UC: Upper Crust; PAAS: Average Post-Archaean Australian Shale; NASC: Average North American Shale Composite; CIA: chemical index of alteration; CIW: chemical index of weathering. 
for CIW and CIA, of $94 \pm 5$ and $85 \pm 4$, respectively, correspond to the samples located in the advanced weathering trend (Fig. 4), i.e., upper portion (surface) samples from Mines 1, 2, 8 and 9.

The indexes do not show any significant changes along both Mine 4 and 7 and stay practically constant with average CIW and CIA values of $69 \pm 1$ and $64 \pm 1$, for Mine 4, respectively, and $69 \pm 2$ and $64 \pm 2$, for Mine 7 , respectively. Thus, in these mines little alteration due to water infiltration and lixiviation, or even by hydrotermalism processes, has occurred.

In Mines 1, 2, 9 and 10 (P10.1, P10.2, P10.3), the CIW and CIA indexes increase from the lower portion towards the upper portion of the profiles, in a range of $65 \pm 15$ to $84 \pm 20$ for CIW and $60 \pm 13$ to $76 \pm 17$ for CIA. The samples from Mines 3 and 6 show a decrease in CIW and CIA indexes towards the upper portion of the profiles, with $63 \pm 13$ index for CIW and $59 \pm 11$ for CIA.

The samples from the middle and upper portion of Mine 1 , as well as the upper portion samples from Mine 2, Mine 5 and Mine 9, and the sample from Mine 8, show the highest indexes of CIW and CIA, which are associated to an elevated weathering degree. In the other hand, the middle and upper portion samples from Mine 3, middle portion samples from Mine 5 and the samples from Mine 10 show the lowest indexes, due to a smaller weathering degree. The remaining samples present similar indexes, which indicates the same degree of weathering in the respective profile portions.

The highest and lowest degrees of weathering can be attributed to the configuration of the profiles within the mines, i.e., lack or presence of a thick surface cover, respectively, depth, time of exposure and local geomorphology, as well as to intrinsic characteristics of these rocks, i.e., permeability and the amount of fractures that can lead to more or less infiltration of rainwater.

In Mine 10, on the contrary of what happens in other mines, the upper portion of the stratigraphic column is still preserved probably due to a strata subsidence, which prevented the carbonates to be weathered and lixiviated from these samples.

Some mines also represent a particular case, as Mines 6 and 7 , that are located in the lowest portion of the stratigraphic column, and Mine 8, which is above the strata, showing because of that an increased weathering index.

The mineralogy in this case isn't representative for a given range of CIW and CIA and cannot be taken as a guide in the weathering evolution, since rocks with distinct compositions present very similar weathering indexes (e.g., groups of samples with the highest and lowest CIW and CIA), rocks with similar mineral composition show completely different weathering indexes (e.g., Mine 5 - P5.2/P5.3 and Mine $9-$ P9.4 samples), and rocks with a mineral

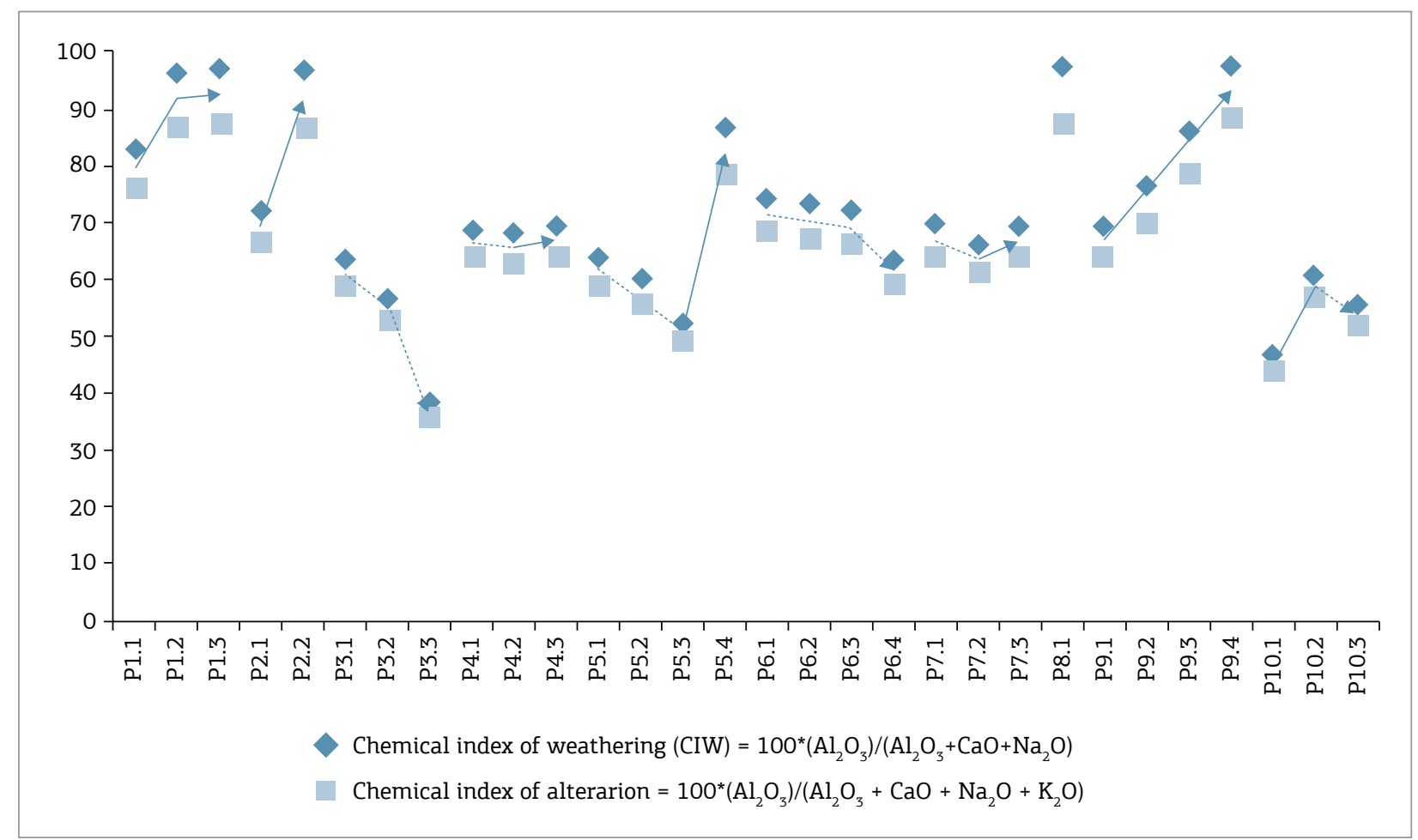

Figure 5. Chemical Index of Weathering, $\mathrm{CIW}=\left[\left(\mathrm{Al}_{2} \mathrm{O}_{3}\right) /\left(\mathrm{Al}_{2} \mathrm{O}_{3}+\mathrm{CaO}+\mathrm{Na}_{2} \mathrm{O}\right)\right]{ }^{*} 100$ and Chemical Index of Alteration, $\mathrm{CIA}=\left[\left(\mathrm{Al}_{2} \mathrm{O}_{3}\right) /\left(\mathrm{Al}_{2} \mathrm{O}_{3}+\mathrm{CaO}+\mathrm{Na}_{2} \mathrm{O}+\mathrm{K}_{2} \mathrm{O}\right)\right] * 100$ for the studied samples. Harnois (1988) and Nesbitt and Young (1982). 
composition more prone to be affected by the weathering effects are in the group of samples with the lowest weathering indexes (i.e., Mine 10, of carbonatic samples, which would be expected to present the highest indexes).

Despite the different location of the profiles within the stratigraphic column of the Corumbataí Formation, apparently they underwent the same erosion/denudation processes, which lead to a similar evolution in terms of decomposition and neoformation of clay minerals, in addition to leaching and concentration of particular elements, allowing the recognition of a weathering pattern between the profiles and mines.

In Mine 10, located in the upper portion of the Corumbataí Formation stratigraphic column, a downward displacement of rock strata prevented the carbonates to be weathered and lixiviated from these profile samples as it would be expected to happen since they are more prone to weather than silicates, therefore being preserved and resulting in the lowest weathering indexes.

\section{TRACE ELEMENT GEOCHEMISTRY}

The trace elements composition of the samples, including REE, is listed in Table 1. The normalized data (by NASC from Gromet et al. 1984 and Taylor \& McLennan 1985; and by $\mathrm{C} 1$ chondrites, Evensen et al. 1978) were plotted in distribution diagrams according to each profile (Figs. 6 and 7).

The distribution of trace elements in one single profile does not show any significant changes from bottom to upper portions, usually with the concentrations of trace elements in the same order of magnitude. Thus, in general, all samples present similar distribution patterns (Fig. 6).

The upper sample from Mine 3 presents a negative anomaly of Cs relative to NASC (Fig. 6), while the bottom and middle samples present a positive anomaly, probably because of its element's mobile character (low charge and large ionic radius) during the weathering processes, being more easily carried out of the system by the weathering fluids.

In Figure 6 one can notice that samples from mines 1, 2, 4, 5,7 and 9 show an increment in REE elements from bottom to upper portions. The chemical indexes (Eu anomaly and light/heavy rations) are listed in Table 2 . The results can be differentiated into two main groups in each profile: lower and upper portion samples (Fig. 7).

The samples from the lower portion of all mines present a fractionation pattern characterized by a continuous depletion in light REE from $\mathrm{La}$ to $\mathrm{Sm}\left(\mathrm{La} / \mathrm{Sm}_{\mathrm{N}}=3.77 \pm 0.27\right)$, followed by a negative anomaly in $\mathrm{Eu}\left(\mathrm{Eu} / \mathrm{Eu}^{*}=0.024 \pm 0.003\right)$ and a regular decreased distribution of heavy REE from $\mathrm{Gd}$ to $\mathrm{Yb}$ $\left(\mathrm{Gd} / \mathrm{Yb}_{\mathrm{N}}=1.51 \pm 0.14\right)$.
The upper portion samples present a similar distribution pattern as described before and REE rations $\mathrm{La} / \mathrm{Sm}_{\mathrm{N}}=3.50 \pm 0.58, \mathrm{Eu} / \mathrm{Eu}^{*}=0.014 \pm 0.003, \mathrm{Gd} / \mathrm{Yb}_{\mathrm{N}}=$ $1.72 \pm 0.19$. However, the concentration of REE in the upper portion $(\Sigma R E E=857 \mathrm{ppm} \pm 99)$ is higher than in the lower portion $(\Sigma \mathrm{REE}=539 \mathrm{ppm} \pm 53)$.

Three of the upper samples present the highest contents in REE (Tab. 1), due to the presence of kaolinite in Mine 9 (P9.4) and Mine 5 (P5.4) and to illite in Mine 2 (P2.2), which can be related to the more advanced weathering state of these samples.

After the analysis of each profile from lower to upper portions, six mining fronts (1, 2, 4, 5, 7 and 9) were identified featuring a REE concentration increase with progressive weathering, i.e., from lower to upper portions (Figs. 7 and 8), probably due to a rapid re-precipitation and loss of major elements that are easily remobilized. In this case, $\mathrm{Na}$ and $\mathrm{Ca}$ oxides usually present a prominent decrease, while other oxides have a slight decrease or maintain a practically constant amount, like $\mathrm{Si}, \mathrm{K}$ and $\mathrm{Mg}$, or an increased amount, like $\mathrm{Al}, \mathrm{Fe}, \mathrm{Ti}, \mathrm{Mn}$ oxides (Moreno et al. 2014).

As the REE are concentrated in the more weathered strata, the sum of REE in each sample, as seen in Figure 8 ( $\Sigma$ REE vs. samples), shows the weathering degree in each profile and how they correlate between the mines. In general, all the upper samples present the highest REE concentrations, with the upper samples from P1, P2, P5 and P9 presenting the sum of REE in the same order of magnitude. The upper samples of $\mathrm{P} 4, \mathrm{P} 7$ and $\mathrm{P} 8$ have correlating values of REE sum. Also, P3, P6 and P10 present corresponding values of REE sum, thus indicating in each case their similarity in terms of weathering evolution.

In three mining fronts (3, 6 and 10), no significant enrichment in REE was noticed along the profiles (Figs. 7 and 8 ), so the fractionation patterns remain in the same order of magnitude, thus implying a higher resistance of these rocks to the weathering, due to the depth of the bench within the profile.

As for Mine 3, there are two benches (benches 1 and 2) underneath the bench where sample P3.1 (bench 3) was collected, which are discarded for being highly altered by hydrothermalism and for its high content in smectites. Above the bench in which sample P3.3 was collected (bench 5), on the top of the profile, there is another bench (bench 6), which was discarded because of its advanced weathering state. Thus, the three benches of Mine 3 that are used as raw material (P3.1, P3.2 and P3.3) correspond to the middle portion of the mine profile, therefore not presenting significative changes in REE composition.

In the front 1 of Mine 6, a different aspect takes place, once the lowest sample (P6.1) presents a increased content 


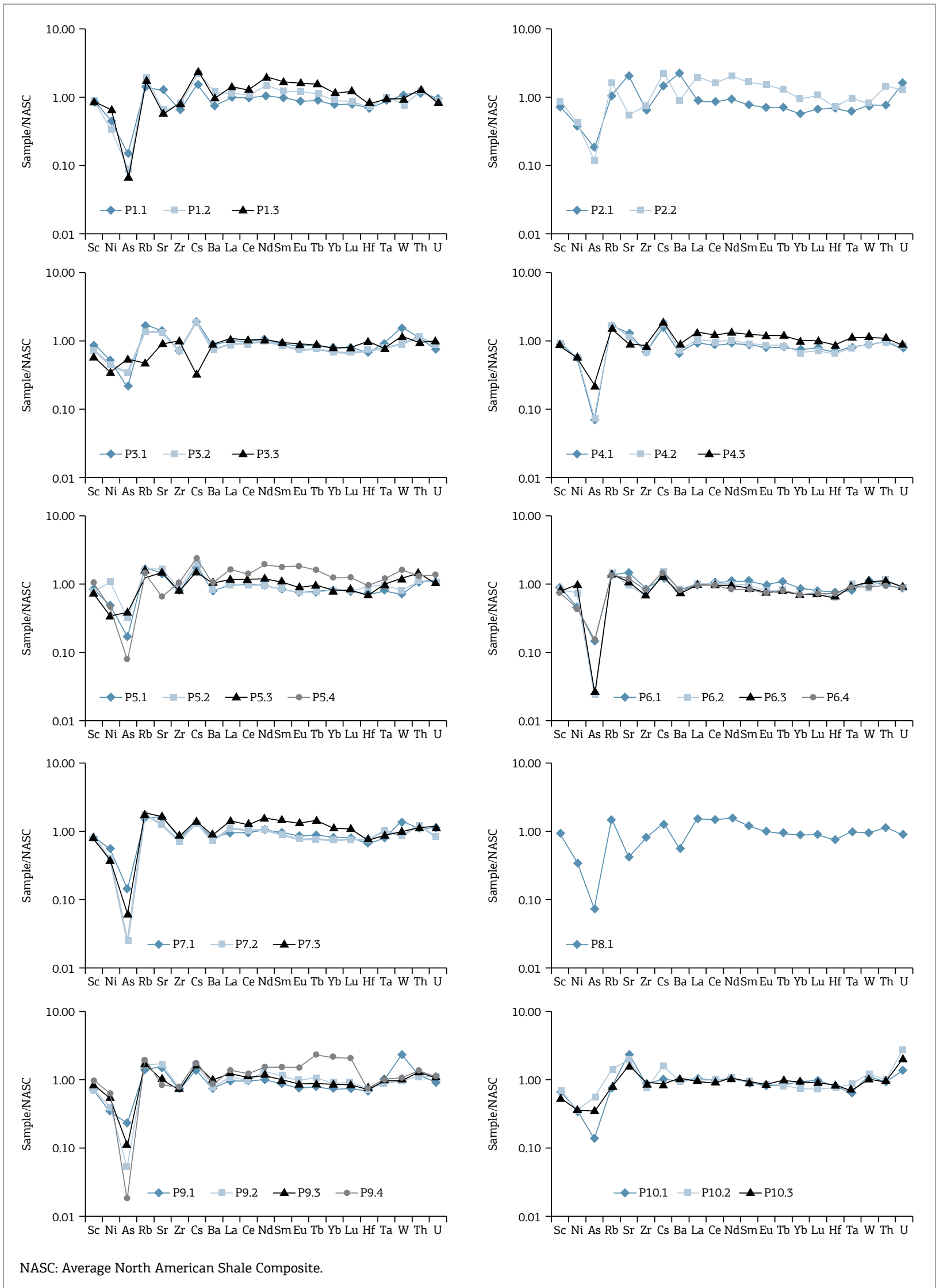

Figure 6. Trace elements distribution for each mine profile. Data normalized by the Average North American Shale Composite (NASC; from Gromet et al., 1984 and Taylor and McLennan, 1985). 

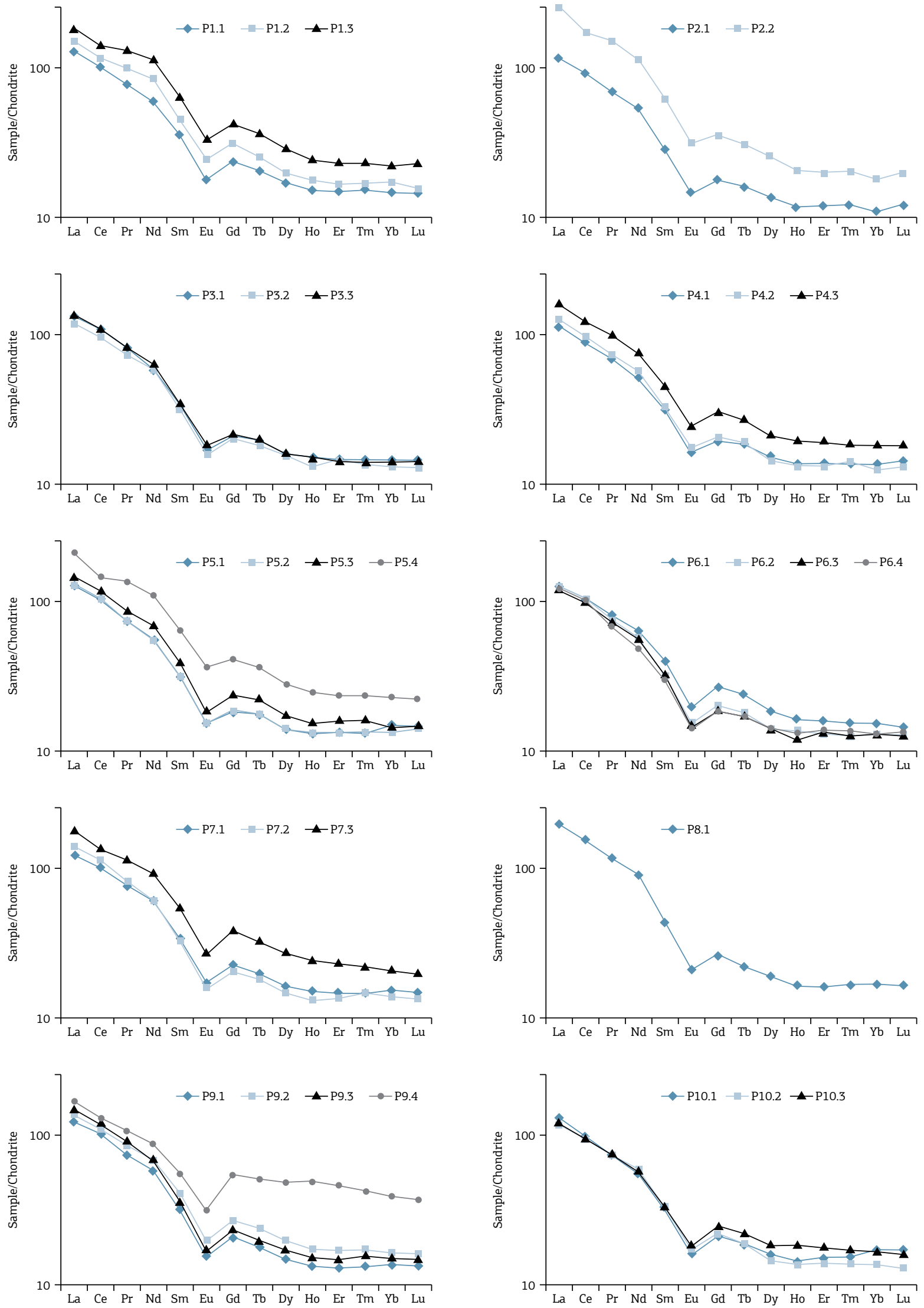

Figure 7. REE patterns for each mine profile. Data normalized by the C1 Chondrite (Evensen et al., 1978). 
in heavy REE (Gd-Lu) when compared to the upper samples (P6.2, P6.3 and P6.4), whose fractionation patterns remain close together in the same order of magnitude. It indicates that in this front a loss of heavy REE occurs, probably due to a preferential transport of these elements because of its stability and low precipitation rates (Formoso et al. 1989).

\section{PROVENANCE}

According to the classification diagram of terrigenous sandstones and shales using $\log \left(\mathrm{Fe}_{2} \mathrm{O}_{3} / \mathrm{K}_{2} \mathrm{O}\right)$ vs. $\log \left(\mathrm{SiO}_{2} / \mathrm{Al}_{2} \mathrm{O}_{3}\right)$ (Herron 1988) and the discriminant function diagram for the provenance signatures of sandstone/mudstone suites using major elements (Roser \& Korsch 1988), the bottom, therefore less weathered, samples of each profile, can be classified as wacke (Fig. 9A), mostly of quartzose sedimentary provenance, with the bottom sample of Mine 10 plotting in the intermediate igneous provenance field (Fig. 9B).

However, the REE and high field strength elements (HFSE) are the elements more suitable for provenance analysis due to the relative more immobile character during weathering, transport, diagenesis and metamorphism. Ratios of both incompatible and compatible elements, such as La/Sc, $\mathrm{Th} / \mathrm{Sc}, \mathrm{Co} / \mathrm{Th}, \mathrm{Cr} / \mathrm{Th}$, and $\mathrm{Eu} / \mathrm{Eu}^{*}$, in siliciclastic sediments, allow to place constraints on the average provenance composition (Cullers et al. 1988, Cullers 1994a, 1995, Wronkiewicz \& Condie 1987, 1989, 1990, Cox et al. 1995). The geochemical differences between elements such as Th and La (indicative of a felsic source) and Sc and $\mathrm{Cr}$ (indicative of

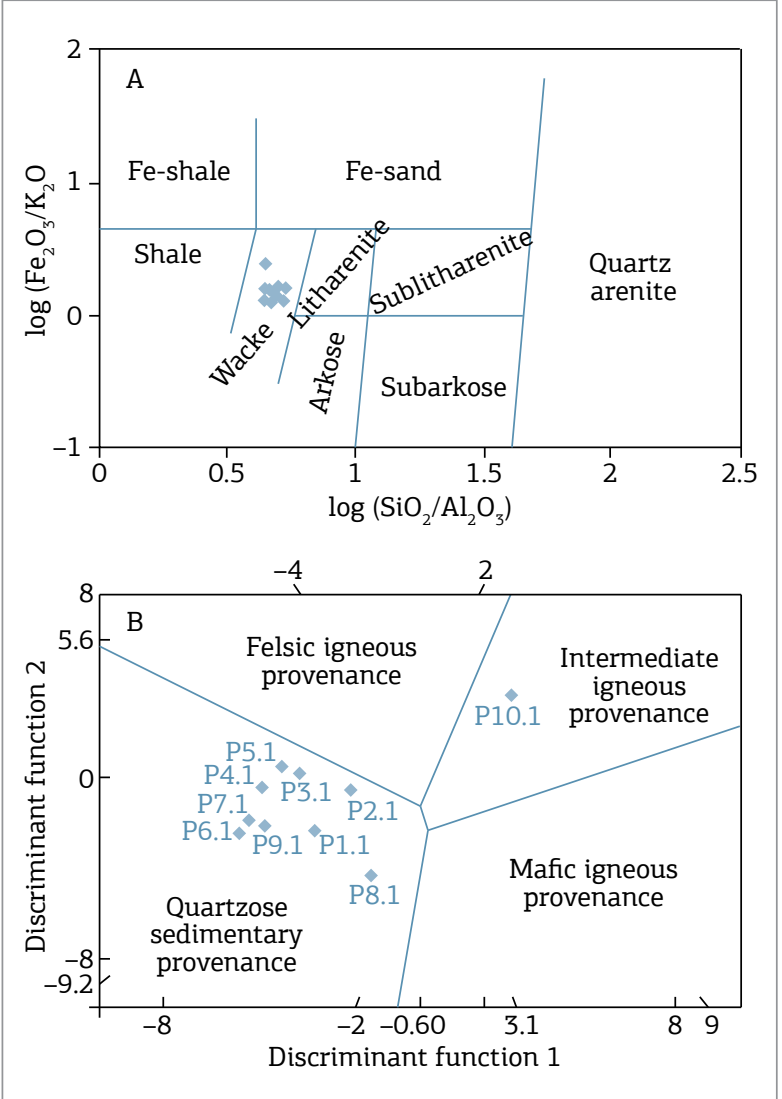

Figure 9. (A) Classification of terrigenous sandstones and shales using $\log \left(\mathrm{Fe}_{2} \mathrm{O}_{3} / \mathrm{K}_{2} \mathrm{O}\right)$ vs. $\log \left(\mathrm{SiO}_{2} / \mathrm{Al}_{2} \mathrm{O}_{3}\right)$ (Herron, 1988). (B) Discriminant function diagram for the provenance signatures of sandstone/mudstone suites using major elements (Roser and Korsch, 1988). In both diagrams only the less weathered samples from the bottom portion of the profiles was used.

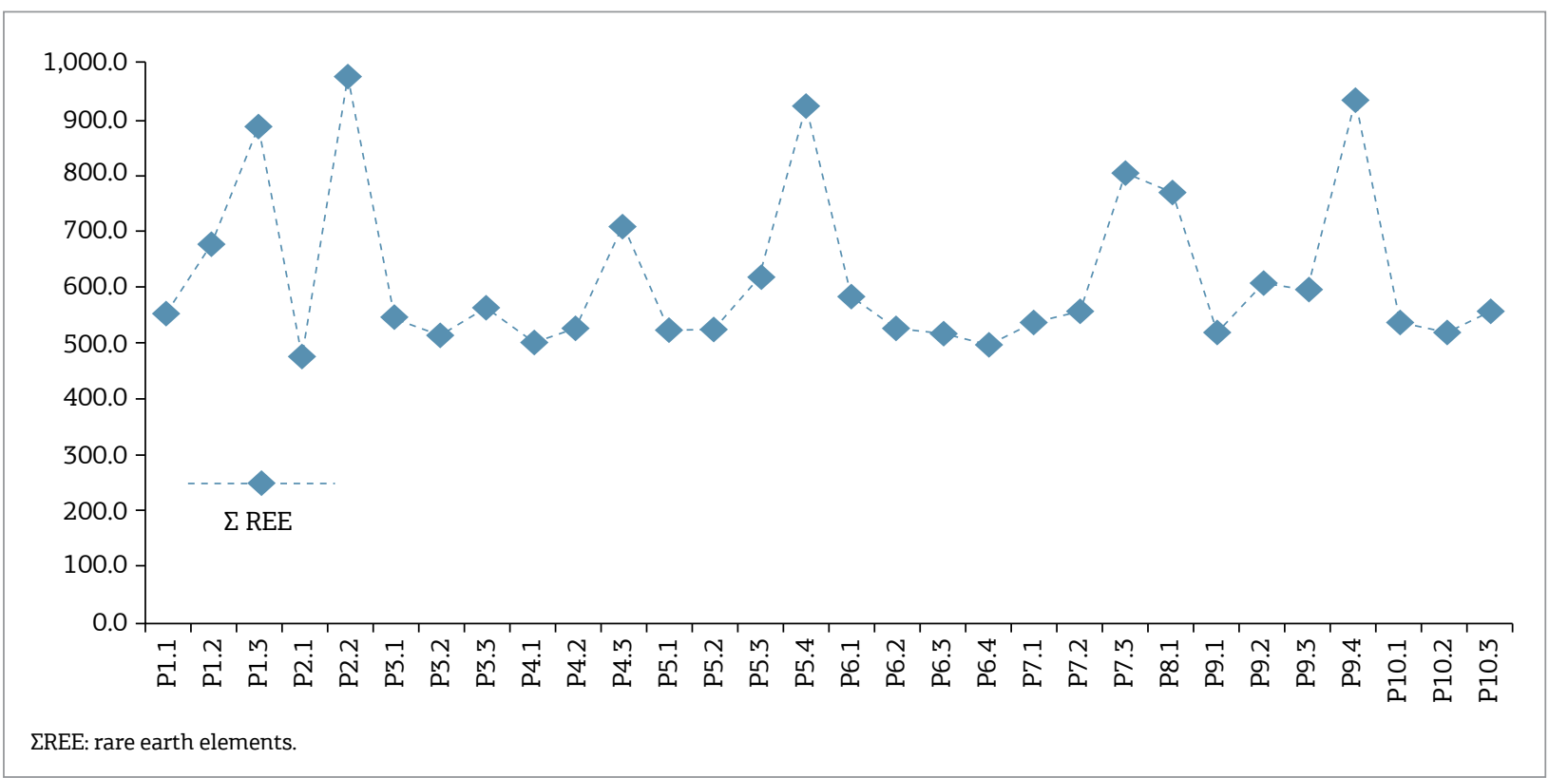

Figure 8. $\Sigma$ REE vs. samples. The diagram shows the concentration increase in the upper portion of each profile, with the exception of Mines 3, 6 and 10 where no significant changes in REE composition occurs. 
a mafic source) have been exploited to distinguish between felsic and mafic provenance by various authors (McLennan 1989, McLennan \& Taylor 1991, McLennan et al. 1980, Wronkiewicz \& Condie 1990). In addition, the mineralogical maturity of the sediments indicated by the proportion of compositionally mature alumina-rich minerals such as kaolinite has a felsic or reworked sedimentary source (Cullers et al. 1975, 1987).

As REE patterns of the source rocks may be preserved in sedimentary rocks (Taylor \& McLennan 1985, Wronkiewicz \& Condie 1987, 1989, Condie et al. 1995, Compton et al. 2003), they were used to infer the source of the SGCP samples. The clayish sedimentary rocks of SGCP present light rare-earth elements (LREEs) enrichment with a high $\mathrm{La} / \mathrm{Sm}_{\mathrm{N}}$ ratio, a negative $\mathrm{Eu}$ anomaly and an almost flat heavy rare earth elements (HREE) pattern with a low $\mathrm{Gd} / \mathrm{Yb}_{\mathrm{N}}$ ratio. These REE patterns are compatible with a more silicic rock source, since they present higher LREE/HREE ratios and negative anomalies, instead of low LREE/HREE ratios and no Eu anomalies presented by basic rock sources (Cullers \& Graf 1983). The negative $\mathrm{Eu}$ anomaly is regarded as evidence for a differentiated source, similar to granite, as pointed out by McLennan (1989), McLennan et al. (1993) and Taylor and McLennan (1985, 1995).

The La and The elements present an immobile character and are more abundant in felsic than basic rocks, whereas Sc and $\mathrm{Co}$ are more concentrated in basic rocks than in felsic rocks (Taylor \& McLennan 1985, Wronkiewicz \& Condie 1987, Condie et al. 1995). The Th/Sc and $\mathrm{La} / \mathrm{Th}$ rations of $1.12 \pm 0.24$ and $2.57 \pm 0.50$, respectively, were used to evaluate the provenance of the samples (Fig. 10). In the $\mathrm{Th} / \mathrm{Sc}$ and $\mathrm{La} / \mathrm{Th}$ diagrams the samples scatter next to the $\mathrm{Th} / \mathrm{Sc}=1$ and $\mathrm{La} / \mathrm{Th}=2.8$ lines, which correspond to the upper continental crust (UC) values, plus all samples present Sc contents below $15 \mathrm{ppm}$ indicate a more felsic component.
In the La-Th-Sc diagram (Fig. 11) that discriminates felsic and basic provenance of fine grained sedimentary rocks (Cullers 1994a, 1994b), the majority of samples fall in a region that indicates a predominant felsic source, which does not discard an intermediate source or a mix of felsic and basic source materials.

Despite intense weathering, shown in Figures 4 and 5 and previously discussed, which affected the contents of most elements, the REE, Th, and Sc apparently remained immobile, allowing the recognition of the source

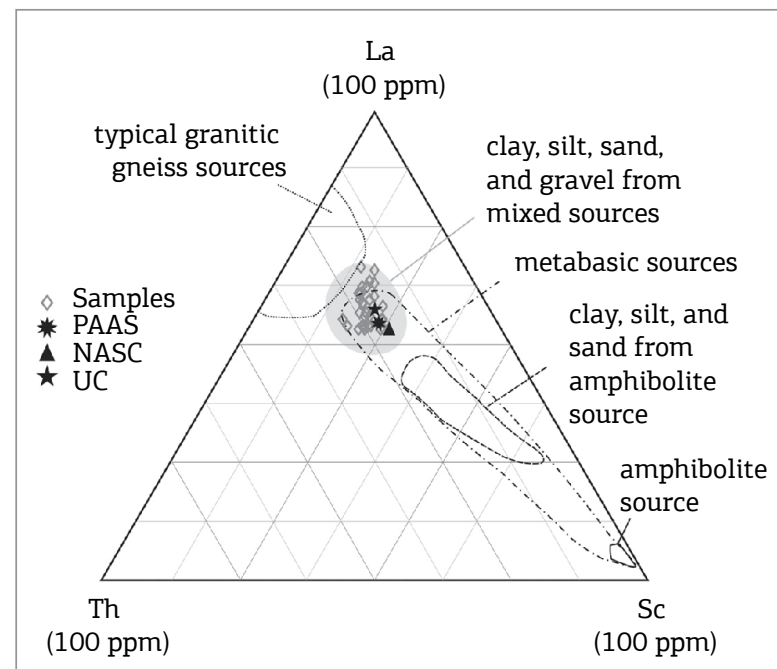

PAAS: Average Post-Archean Australian Shale; NASC: Average North American Shale Composite; UC: Upper Crust.

Figure 11. Ternary plot of La-Th-Sc for the Corumbataí Formation samples after Cullers (1994). The Upper Crust (UC; Taylor and McLennan, 1985), Average Post-Archean Australian Shale (PAAS; Taylor and McLennan, 1985) and Average North American Shale Composite (NASC; Gromet et al., 1984 and Taylor and McLennan, 1985) are plotted for comparisons.
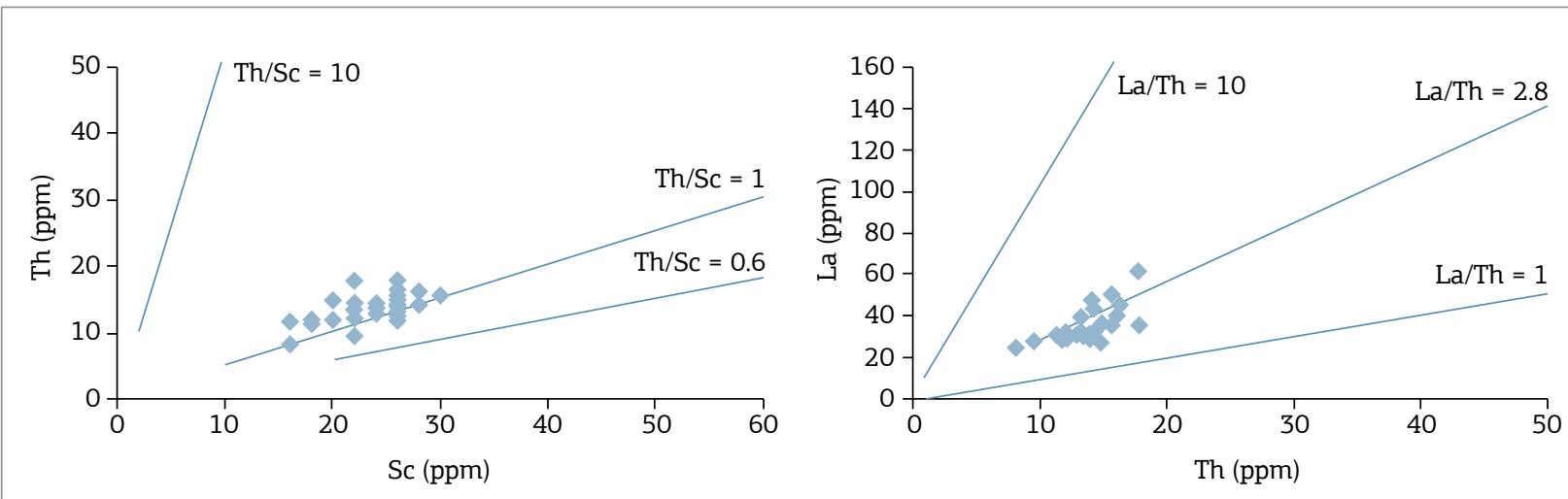

Figure 10. Incompatible elements plot for the studied samples. Th versus Sc, where Th/Sc $=1$ ratio is that of the Upper Crust. La versus Th, where La/Th $=2.8$ ratio is that of Upper Crust (data from Taylor and McLennan, 1985). 
area composition. Also, the slight differences among the REE patterns probably do not reflect changes in source area composition, but are likely due to variations in mineralogy and weathering (Nyakairu \& Koeberl 2001, Mahjoor et al. 2009).

\section{CONCLUSIONS}

We analyzed 30 samples from 10 mining fronts of clayish rocks of the Corumbataí Formation, in the region of the SGCP (São Paulo, Brazil), in order to determine their mineralogical and chemical compositions, REE characteristics, weathering trends and provenance. XRD analysis shows that the sedimentary rocks are mainly composed by illite, quartz and albite, being differentiated by the content of other clayish minerals as kaolinite and smectite, or by the presence of minor minerals such as calcite, microcline, hematite and/or chlorite.

The studied profiles from the Corumbataí Formation in the SGCP region show diagnostic geochemical signatures that can be correlated from mine to mine, i.e., the constant $\mathrm{MgO} / \mathrm{K}_{2} \mathrm{O}$ ratio (consequence of smectite and illite neoformation) and the variation of other major and minor oxides concentrations, the recognition of three main groups of samples that follow the compositions of UC (G1), NASC and PAAS (G2) and one of an advanced weathering condition (G3), the similar groups of CIW and CIA and the immobile character of REE, Th, and Sc.
The claystones of the SGCP region were intensely affected by the weathering processes, which remobilized alkali and alkali earth elements towards the upper portion of each mine profile and re-precipitated and/or relatively concentrated REE in the uppermost samples of the studied profiles.

The erosion/denudation processes were apparently the same among the studied profiles from the SGCP region, allowing the recognition of a similar weathering pattern among the mines, despite its location within the stratigraphic column of the Corumbataí Formation. A downward displacement of rock strata was especially important, since it prevented the carbonates from Mine 10 to be weathered and lixiviated from it, leading to the lowest CIW and CIA indexes, the contrary of what would be expected, since they are more prone to be weathered than the silicate samples.

Mineralogical and geochemical data here reported suggest that the provenance of the sedimentary rocks was dominated by felsic sources, but intermediate or mixed sources cannot be discarded. The chemical and mineralogical aspects observed for the clayish samples of the SGCP are practically uniform along the studied profiles and mines, which allow them to be used as raw material by the industries of the SGCP region.

\section{ACKNOWLEDGEMENTS}

We are grateful to the anonymous colleagues for helpful and constructive reviews, which led to improvements in the manuscript.

\section{REFERENCES}

Azzi A.A., Osacký M., Uhlík P., Čaplovičová M., Zanardo A., Madejová J. 2016. Characterization of clays from the Corumbataí formation used as raw material for ceramic industry in the Santa Gertrudes district, São Paulo, Brazil. Applied Clay Science, 132-133:232-242.

Costa M.N.S. 2006. Diagênese e alteração hidrotermal em rochas sedimentares da Formação Corumbataí, Permiano Superior, Mina Granusso, Cordeirópolis/SP. PhD Thesis, Universidade Estadual Paulista, Rio Claro, São Paulo, 140 p.

Compton J.S., White R.A., Smith M. 2003. Rare earth element behavior in soils and salt pan sediments of a semi-arid granitic terrain in the Western Cape, South Africa. Chemical Geology, 201(3-4):239-255.

Condie K.C., Dengate J., Cullers R.L. 1995. Behavior of rare earth elements in a paleoweathering profile on granodiorite in the Front Range, Colorado, USA. Geochimica et Cosmochimica Acta, 59(2):279-294

Cox R., Lowe D.R., Cullers R.L. 1995. The influence of sediment recycling and basement composition on evolution of mudrock chemistry in the southwestern United States. Geochimica et Cosmochimica Acta, 59(14):2919-2940.

Cullers R.L. 1994a. The controls on the major and trace element variation of shales, siltstones, and sandstones of PennsylvanianPermian age from uplifted continental blocks in Colorado to platform sediment in Kansas, USA. Geochimica et Cosmochimica Acta, 58(22):4955-4972.

Cullers R.L. 1994b. The chemical signature of source rocks in size fractions of Holocene stream sediment derived from metamorphic rocks in the Wet Mountains region, Colorado, USA. Chemical Geology, 113(3-4):327-343.

Cullers R.L. 1995. The controls on the major- and trace-element evolution of shales, siltstones and sandstones of Ordovician to tertiary age in the Wet Mountains region, Colorado, USA. Chemical Geology, 123(1-4):107-131

Cullers R.L., Barrett T., Carlson R., Robinson B. 1987. Rare-earth element and mineralogic changes in Holocene soil and stream sediment: A case study in the Wet Mountains, Colorado, USA. Chemical Geology, 63(3-4):275-297.

Cullers R.L., Basu A., Suttner L.J. 1988. Geochemical signature of provenance in sand-size material in soils and stream sediments near the Tobacco Root batholith, Montana, USA. Chemical Geology, 70(4):335-348

Cullers R.L., Chaudhuri S., Arnold B., Lee M., Wolf Jr. C.W.W. 1975. Rare earth distributions in clay minerals and in clay-sized fraction of the Lower Permian Havensville and Eskridge shales of Kansas and Oklahoma. Geochimica et Cosmochimica Acta, 39(12):1691-1703. 
Cullers R.L. \& Graf J. 1983. Rare earth elements in igneous rocks of the continental crust: intermediate and silicic rocks, ore petrogenesis. In: Henderson P. (ed.) Rare-Earth Geochemistry. Amsterdam, Elsevier, p. 275-312.

Evensen N.M., Hamilton P.J., O'nions R.K. 1978. Rare-earth abundances in chondritic meteorites. Geochimica et Cosmochimica Acta, 42(8):1199-1212.

Formoso M.L.L., Melfi A.J., Kronberg B.I. 1989. Comportamento dos elementos terras raras no intemperismo tropical. In: Formoso M.L.L., Nardi L.V.S., Hartmann L.A. (eds.). Geoquímica dos elementos terras raras no Brasil. Porto Alegre, Companhia de Pesquisa de Recursos Minerais (CPRM) / Departamento Nacional de Produção Mineral (DNPM), p. 109-120.

Fúlfaro V.J. \& Bjornberg A. 1983. Solos do Interior de São Paulo. São Paulo, Editora EDUSP, 1-39 p.

Gromet L.P., Dymek R.F., Haskin L.A., Korotev R.L. 1984. The "North American shale composite": Its compilation, major and trace element characteristics. Geochimica et Cosmochimica Acta, 48:2469-2482.

Harnois L. 1988. The CIW index: A new chemical index of weathering. Sedimentary Geology, 55(3-4):322

Herron M.M. 1988. Geochemical classification of terrigenous sands and shales from core or log data. Journal of Sedimentary Research, 58(5):820-829.

Mahjoor A.S., Karimi M., Rastegarlari A. 2009. Mineralogical and geochemical characteristics of clay deposits from South Abarkouh District of Clay Deposit (Central Iran) and their applications. Journal of Applied Sciences, 9(4):601-614

McLennan S.M. 1989. Rare earth elements in sedimentary rocks; influence of provenance and sedimentary processes. Reviews in Mineralogy, 21(1):169-200

McLennan S.M., Hemming S., McDaniel D.K., Hanson G.N. 1993. Geochemical approaches to sedimentation, provenance, and tectonics. Geological Society of America Special Papers, Processes Controlling the Composition of Clastic Sediments, 284:21-40.

McLennan S.M., Nance W.B., Taylor S.R. 1980. Rare earth elementthorium correlations in sedimentary rocks, and the composition of the continental crust. Geochimica et Cosmochimica Acta, 44(11):1833-1839.

McLennan S.M. \& Taylor S.R. 1991. Sedimentary rocks and crustal evolution: Tectonic setting and secular trends. The Journal of Geology, 99(1):1-21.

Milani E.J., França A.B., Schneider R.L. 1994. Bacia do Paraná. Boletim de Geociências da Petrobrás, 8:69-72.

Moreno M.M.T., Bartolomeu D., Lima R.H.C. 2009. Análise do comportamento de queima de argilas e formulações para revestimento cerâmico. Cerâmica, 55(335):286-295.

Moreno M.M.T., Da Rocha R.R., Godoy L.H. 2014. Major elements geochemistry of sedimentary rocks from Corumbataí Formation, Santa Gertrudes Ceramic Pole, São Paulo, Brazil. Geomaterials, 4(1):11-17.

Nesbitt H.W. \& Young G.M. 1982. Early Proterozoic climates and plate motions inferred from major element chemistry of lutites. Nature, 299:715-717.
Nesbitt H.W. \& Young G.M. 1984. Prediction of some weathering trends of plutonic and volcanic rocks based on thermodynamic and kinetic considerations. Geochimica et Cosmochimica Acta, 48(7):1523-1534.

Nesbitt H.W. \& Young G.M. 1989. Formation and diagenesis of weathering profiles. The Journal of Geology, 97(2):129-147.

Nyakairu G.W.A. \& Koeberl C. 2001. Mineralogical and chemical composition and distribution of rare earth elements in clay-rich sediments from central Uganda. Chemical Journal, 35(1):13-28.

Penteado M.M. 1976. Geomorfologia do Setor Centro-Ocidental da Depressão Periférica Paulista. PhD Thesis, Universidade de São Paulo, $86 \mathrm{p}$.

Roser B.P. \& Korsch R.J. 1988. Provenance signatures of sandstonemudstone suites determined using discriminant function analysis of major element data. Chemical Geology, 67(1-2):119-139.

Taylor S.R. \& McLennan S.H. 1985. The Continental Crust: Its Composition and Evolution. Blackwell. Oxford, 312 p.

Taylor S.R. \& McLennan S.M. 1995. The geochemical evolution of the continental crust. Reviews of Geophysics, 33(2):241-265.

Thiry M., Carrillo N., Franke C., Martineau N. 2013. Technique de preparation des mineraux argileux en vue de l'analyse par diffraction des Rayons $\mathrm{X}$ et introduction a l'interprétation des diagrammes. Geosciences, hal-00872214(1). Available at: https://hal-minesparistech.archives-ouvertes.fr/hal-00872214 [cited at Aug. 2017].

White I.C. 1908. Relatório Final sobre as medidas de carvão e rochas associadas do Sul do Brasil. Rio de Janeiro, Relatório da Comissão das Minas de Carvão de Pedra do Brasil, 300 p.

Wronkiewicz D.J. \& Condie K.C. 1987. Geochemistry of Archean shales from the Witwatersrand Supergroup, South Africa: Sourcearea weathering and provenance. Geochimica et Cosmochimica Acta, 51(9):2401-2416.

Wronkiewicz D.J. \& Condie K.C. 1989. Geochemistry and provenance of sediments from the Pongola Supergroup, South Africa: Evidence for a 3.0-Ga-old continental craton. Geochimica et Cosmochimica Acta, 53(7):1537-1549.

Wronkiewicz D.J. \& Condie K.C. 1990. Geochemistry and mineralogy of sediments from the Ventersdorp and Transvaal Supergroups, South Africa: Cratonic evolution during the early Proterozoic. Geochimica et Cosmochimica Acta, 54(2):343-354.

Zalán P.V., Wolff S., Astolfi M.A.M., Vieira I.S., Conceição J.C.J., Appi V.T., Neto E.V.S., Cerqueira J.R., Marques A. 1990. The Paraná Basin, Brazil. In: Leighton M.W., Kolata D.R., Oltz D.F., Eidel J.J. Interior cratonic hasins. Tulsa, American Association of Petroleum Geologists Memoir, 51, p. 681-708.

Zanardo A., Montibeller C.C., Navarro G.R.B., Moreno M.M.T., Da Rocha R.R., Del Roveri C., Azzi A.A. 2016. Formação Corumbataí na região de Rio Claro/SP: petrografia e implicações genéticas. Universidade Estadual Paulista, São Paulo, Geociências, 35:322-345. 